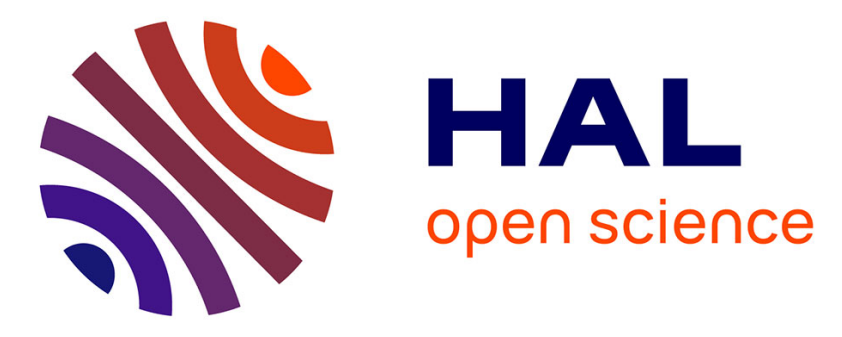

\title{
Iron isotope fractionation in planetary crusts
}

Kun Wang, Frédéric Moynier, Nicolas Dauphas, Jean-Alix J-A Barrat, Paul Craddock, Corliss K. Sio

\section{To cite this version:}

Kun Wang, Frédéric Moynier, Nicolas Dauphas, Jean-Alix J-A Barrat, Paul Craddock, et al.. Iron isotope fractionation in planetary crusts. Geochimica et Cosmochimica Acta, 2012, 89, pp.31-45. 10.1016/j.gca.2012.04.050 . insu-00714561

\section{HAL Id: insu-00714561 https://hal-insu.archives-ouvertes.fr/insu-00714561}

Submitted on 25 Feb 2013

HAL is a multi-disciplinary open access archive for the deposit and dissemination of scientific research documents, whether they are published or not. The documents may come from teaching and research institutions in France or abroad, or from public or private research centers.
L'archive ouverte pluridisciplinaire HAL, est destinée au dépôt et à la diffusion de documents scientifiques de niveau recherche, publiés ou non, émanant des établissements d'enseignement et de recherche français ou étrangers, des laboratoires publics ou privés. 


\title{
Iron isotope fractionation in planetary crusts
}

\author{
Kun Wang ${ }^{\mathrm{a}, *}$, Frédéric Moynier ${ }^{\mathrm{a}}$, Nicolas Dauphas ${ }^{\mathrm{b}}$, Jean-Alix Barrat ${ }^{\mathrm{c}}$, \\ Paul Craddock ${ }^{\mathrm{b}}$, Corliss K. Sio ${ }^{\mathrm{b}}$ \\ ${ }^{a}$ Department of Earth and Planetary Sciences, McDonnell Center for the Space Sciences, Washington University in St. Louis, \\ One Brookings Drive, St. Louis, MO 63130, United States \\ ${ }^{\mathrm{b}}$ Origin Laboratory, Department of the Geophysical Sciences, Enrico Fermi Institute, The University of Chicago, \\ 5734 South Ellis Avenue, Chicago, IL 60637, United States \\ ${ }^{\mathrm{c}}$ Université de Brest, CNRS UMR 6538 (Domaines Océaniques), I.U.E.M., Place Nicolas Copernic, 29280 Plouzané Cedex, France
}

Received 10 November 2011; accepted in revised form 23 April 2012; available online 27 April 2012

\begin{abstract}
We present new high precision iron isotope data ( $\delta^{56} \mathrm{Fe}$ vs. IRMM-014 in per mil) for four groups of achondrites: one lunar meteorite, 11 martian meteorites, 32 howardite-eucrite-diogenite meteorites (HEDs), and eight angrites. Angrite meteorites are the only planetary materials, other than Earth/Moon system, significantly enriched in the heavy isotopes of Fe compared to chondrites (by an average of $+0.12 \%$ in $\delta^{56} \mathrm{Fe}$ ). While the reason for such fractionation is not completely understood, it might be related to isotopic fractionation by volatilization during accretion or more likely magmatic differentiation in the angrite parent-body. We also report precise data on martian and HED meteorites, yielding an average $\delta^{56} \mathrm{Fe}$ of $0.00 \pm 0.01 \%$. Stannern-trend eucrites are isotopically heavier by $+0.05 \%$ in $\delta^{56} \mathrm{Fe}$ than other eucrites. We show that this difference can be ascribed to the enrichment of heavy iron isotopes in ilmenite during igneous differentiation. Preferential dissolution of isotopically heavy ilmenite during remelting of eucritic crust could have generated the heavy iron isotope composition of Stannern-trend eucrites. This supports the view that Stannern-trend eucrites are derived from main-group eucrite source magma by assimilation of previously formed asteroidal crust.

These new results show that iron isotopes are not only fractionated in terrestrial and lunar basalts, but also in two other differentiated planetary crusts. We suggest that igneous processes might be responsible for the iron isotope variations documented in planetary crusts.
\end{abstract}

(C) 2012 Elsevier Ltd. All rights reserved.

\section{INTRODUCTION}

Iron is the ninth most abundant element in the Solar System, and the second most abundant element in the Earth and other terrestrial planetary bodies. Iron can behave as a siderophile (metal-loving), lithophile (rock-loving) and chalcophile (sulfur-loving) element and is ubiquitous in Solar System planetary bodies. On Earth, it is a major element in the core, the mantle and the crust (Allègre et al., 1995; Rudnick and Gao, 2003). In the past decade, the development of high-resolution Multi-Collector Inductively-

\footnotetext{
* Corresponding author.

E-mail address: wangkun@wustl.edu (K. Wang).
}

Coupled-Plasma Mass-Spectrometers (MC-ICP-MS) has allowed measurements of iron isotope composition at high precision (Belshaw et al., 2000; Zhu et al., 2001; Weyer and Schwieters, 2003; Dauphas et al., 2009b; Millet et al., 2012). Following this improvement, small yet resolvable iron isotopic variations in igneous rocks have been discovered (Poitrasson et al., 2004; Weyer et al., 2005; Schoenberg and von Blanckenburg, 2006; Weyer and Ionov, 2007; Heimann et al., 2008; Teng et al., 2008, 2011; Dauphas et al., 2009a; Weyer and Seitz, 2012).

Mid-ocean ridge basalts (MORBs), oceanic island basalts (OIBs), and continental basalts are enriched in heavy iron isotopes by $\sim+0.1 \%$ when compared to chondrites; while martian and 4-Vesta (HED) meteorites all have 
similar iron isotope compositions to chondrites (Poitrasson et al., 2004; Weyer et al., 2005; Anand et al., 2006; Schoenberg and von Blanckenburg, 2006; Craddock and Dauphas, 2011). Three mechanisms have been proposed to explain this difference: (1) Planetary accretion: Heavy iron isotopes were enriched on the Earth and Moon by evaporative kinetic isotope fractionation during the giant impact that formed the Moon (Poitrasson et al., 2004). In this context, one of the difficulties is to explain why potassium, which is highly volatile, is not isotopically fractionated in the EarthMoon system (Humayun and Clayton, 1995). Poitrasson et al. (2004) proposed that $\mathrm{Fe}$ was evaporated from core material as metal while $\mathrm{K}$ was evaporated as oxides. In that case, Fe would be more volatile than $\mathrm{K}$ and could be isotopically more fractionated. However, lunar mare basalts (low-Ti vs. high-Ti) have large variable iron isotope compositions and the $\delta^{56} \mathrm{Fe}$ value of the bulk Moon is not well known (Liu et al., 2010), which provides little constraint on the evaporative isotope fractionation hypothesis. (2) Core-mantle segregation: At ultra high-pressures (>100 GPa) relevant to terrestrial core-mantle boundary conditions, Polyakov (2009) suggested based on nuclear resonant inelastic X-ray scattering data that a detectable fractionation between metallic and silicate phases should be present, which could explain the heavy iron isotope composition of terrestrial silicate rocks. While the approach used by Polyakov (2009) is sound, the results are highly dependent on the high-energy tails of the phonon density of states, which are highly uncertain for high-pressure minerals. In contrast to this hypothesis, laboratory experiments at temperatures and pressures applicable to differentiation of parent bodies to achondrites have shown no iron isotope fractionation between metal and silicate minerals (Poitrasson et al., 2009; Hin et al., 2010). (3) Crust formation: It has been widely observed that iron isotopes could be fractionated during various magmatic differentiation processes of the terrestrial crust, such as partial melting, mineral frac- tionation and fluids exsolution (Williams et al., 2004; Poitrasson and Freydier, 2005; Weyer and Ionov, 2007; Teng et al., 2008; Schuessler et al., 2009). Island arc basalts also show iron isotope fractionation that may be related to the degree of partial melting (Dauphas et al., 2009a). During partial melting, $\mathrm{Fe}(\mathrm{III})$ is more incompatible in olivine and pyroxene than $\mathrm{Fe}(\mathrm{II})$ is. Both theoretical calculations and experimental determinations show that $\mathrm{Fe}(\mathrm{III})$-bearing phases tend to be enriched in the heavy isotopes of iron compared to $\mathrm{Fe}(\mathrm{II})$-bearing phases (Polyakov and Mineev, 2000; Schauble et al., 2001; Schuessler et al., 2007; Shahar et al., 2008). Such equilibrium isotope fractionation between $\mathrm{Fe}(\mathrm{III})$ and $\mathrm{Fe}(\mathrm{II})$ may explain, at least in part, the heavy iron isotope composition of MORBs and OIBs relative to that of chondrites and other planetary basalts (Dauphas et al., 2009a). Indeed, terrestrial basalts are formed under more oxidizing conditions than martian meteorites or HEDs (McCammon, 2005; Wadhwa, 2008).

Achondrites are samples from differentiated planetary bodies formed under a variety of conditions. Here, we report high-precision iron isotope compositions of several classes of achondrites from at least four different parent bodies, including a lunar meteorite, martian meteorites, howardite-eucrite-diogenite meteorites (HEDs) and angrites, and discuss the implications regarding the conditions relevant to the differentiation of the parent-bodies of these meteorites.

\section{SAMPLES AND METHODS}

\subsection{Sample descriptions}

All samples, their classifications and weathering conditions (if available) are listed in Table 1. Well-characterized terrestrial geostandards were analyzed to assess the quality of the measurements and to provide a basis for inter-laboratory comparisons. BCR-2 is a continental flood basalt

Table 1

Iron isotope compositions of Earth/Moon rocks and achondrites.

\begin{tabular}{|c|c|c|c|c|c|c|c|c|c|}
\hline Sample & Type & $\begin{array}{l}\text { Fall/find } \\
\text { (weathering) }\end{array}$ & Chemistry & $\begin{array}{l}\mathrm{Fe} \\
\text { wt. } \%\end{array}$ & $\delta^{56} \mathrm{Fe}$ & $\begin{array}{l}95 \% \\
\text { c. }^{2}{ }^{a}\end{array}$ & $\delta^{57} \mathrm{Fe}$ & $\begin{array}{l}95 \% \\
\text { c. }^{\mathrm{a}}{ }^{\mathrm{a}}\end{array}$ & $n$ \\
\hline \multicolumn{10}{|l|}{ Earth/Moon } \\
\hline AGV-1 \#1 & Andesite & & Routine $^{\mathrm{b}}$ & & 0.081 & 0.034 & 0.133 & 0.049 & 8 \\
\hline AGV-1 \#2 & Andesite & & Routine $^{\mathrm{b}}$ & & 0.077 & 0.045 & 0.105 & 0.058 & 8 \\
\hline AGV-1 \#3 & Andesite & & Routine $^{\mathrm{b}}$ & & 0.081 & 0.046 & 0.134 & 0.066 & 9 \\
\hline AGV-1 \#4 & Andesite & & Routine $^{\mathrm{c}}$ & & 0.106 & 0.031 & 0.157 & 0.039 & 11 \\
\hline AGV-1 (average) & & & & & 0.089 & 0.019 & 0.138 & 0.025 & \\
\hline AGV-2 \#1 & Andesite & & Routine $^{\mathrm{b}}$ & 4.6 & 0.094 & 0.038 & 0.137 & 0.057 & 9 \\
\hline AGV-2 \#2 & Andesite & & Routine $^{\mathrm{b}}$ & & 0.083 & 0.035 & 0.094 & 0.057 & 8 \\
\hline AGV-2 \#3 & Andesite & & Routine $^{c}$ & & 0.100 & 0.031 & 0.167 & 0.040 & 10 \\
\hline AGV-2 (average) & & & & & 0.093 & 0.020 & 0.141 & 0.028 & \\
\hline BCR-2 \#1 & Basalt & & Routine $^{\mathrm{b}}$ & 11.0 & 0.063 & 0.032 & 0.080 & 0.046 & 9 \\
\hline BCR-2 \#2 & Basalt & & Routine $^{\mathrm{b}}$ & 11.0 & 0.052 & 0.033 & 0.110 & 0.058 & 9 \\
\hline BCR-2 \#3 & Basalt & & Routine $^{c}$ & & 0.089 & 0.031 & 0.110 & 0.039 & 11 \\
\hline BCR-2 (average) & & & & & 0.069 & 0.018 & 0.100 & 0.026 & \\
\hline BHVO-2 \#1 & Basalt & & Routine $^{\mathrm{c}}$ & & 0.116 & 0.038 & 0.164 & 0.057 & 4 \\
\hline
\end{tabular}


Table 1 (continued)

\begin{tabular}{|c|c|c|c|c|c|c|c|c|c|}
\hline Sample & Type & $\begin{array}{l}\text { Fall/find } \\
\text { (weathering) }\end{array}$ & Chemistry & $\begin{array}{l}\mathrm{Fe} \\
\text { wt. } \%\end{array}$ & $\delta^{56} \mathrm{Fe}$ & $\begin{array}{l}95 \% \\
\text { c.i. }^{a} \\
\end{array}$ & $\delta^{57} \mathrm{Fe}$ & $\begin{array}{l}95 \% \\
\text { c.i. }^{a}\end{array}$ & $n$ \\
\hline BHVO-2 \#2-1 & Basalt & & Routine $^{\mathrm{d}}$ & 6.3 & 0.099 & 0.030 & 0.155 & 0.044 & 9 \\
\hline BHVO-2 \#2-2 & Basalt & & Long $^{\mathrm{d}}$ & 7.9 & 0.096 & 0.030 & 0.160 & 0.053 & 9 \\
\hline BHVO-2 \#2-3 & Basalt & & UTEVA $^{\mathrm{d}}$ & 8.7 & 0.101 & 0.030 & 0.166 & 0.044 & 9 \\
\hline $\begin{array}{l}\text { BHVO-2 \#3-1 } \\
(+\mathrm{V}+\mathrm{Cr})^{\mathrm{j}}\end{array}$ & Basalt & & Routine $^{\mathrm{d}}$ & 6.7 & 0.108 & 0.030 & 0.161 & 0.044 & 9 \\
\hline $\begin{array}{l}\text { BHVO-2 \#3-2 } \\
(+\mathrm{V}+\mathrm{Cr})^{\mathrm{j}}\end{array}$ & Basalt & & Long $^{\mathrm{d}}$ & 8.5 & 0.098 & 0.030 & 0.154 & 0.044 & 9 \\
\hline $\begin{array}{l}\text { BHVO-2 \#3-3 } \\
(+\mathrm{V}+\mathrm{Cr})^{\mathrm{j}}\end{array}$ & Basalt & & UTEVA $^{\mathrm{d}}$ & 9.0 & 0.098 & 0.030 & 0.154 & 0.044 & 9 \\
\hline BHVO-2 (average) & & & & & 0.102 & 0.012 & 0.159 & 0.018 & \\
\hline $\begin{array}{l}\text { BIR-1 \#1 } \\
\text { BIR-1 \#2 } \\
\text { BIR-1 \#3 } \\
\text { BIR-1 \#4 } \\
\text { BIR-1 (average) }\end{array}$ & $\begin{array}{l}\text { Basalt } \\
\text { Basalt } \\
\text { Basalt } \\
\text { Basalt }\end{array}$ & & $\begin{array}{l}\text { Routine }^{\mathrm{b}} \\
\text { Routine }^{\mathrm{b}} \\
\text { Routine }^{\mathrm{b}} \\
\text { Routine }^{\mathrm{c}}\end{array}$ & $\begin{array}{l}7.7 \\
7.7\end{array}$ & $\begin{array}{l}0.044 \\
0.038 \\
0.020 \\
0.068 \\
0.043\end{array}$ & $\begin{array}{l}0.030 \\
0.038 \\
0.032 \\
0.031 \\
0.016\end{array}$ & $\begin{array}{l}0.070 \\
0.047 \\
0.019 \\
0.070 \\
0.058\end{array}$ & $\begin{array}{l}0.049 \\
0.075 \\
0.059 \\
0.040 \\
0.026\end{array}$ & $\begin{array}{r}9 \\
8 \\
8 \\
10\end{array}$ \\
\hline $\begin{array}{l}\text { NMNH 116852-1 \#1-1 } \\
\text { NMNH 116852-1 \#1-2 } \\
\text { NMNH 116852-1 (averag }\end{array}$ & $\begin{array}{l}\text { Basalt } \\
\text { Basalt } \\
\text { ge) }\end{array}$ & & $\begin{array}{l}\text { Routine }^{\mathrm{d}} \\
\text { UTEVA }^{\mathrm{d}}\end{array}$ & $\begin{array}{l}3.7 \\
6.4\end{array}$ & $\begin{array}{l}-0.148 \\
-0.081 \\
-0.115\end{array}$ & $\begin{array}{l}0.035 \\
0.035 \\
0.025\end{array}$ & $\begin{array}{l}-0.223 \\
-0.134 \\
-0.179\end{array}$ & $\begin{array}{l}0.050 \\
0.050 \\
0.035\end{array}$ & $\begin{array}{l}9 \\
9\end{array}$ \\
\hline $\begin{array}{l}\text { NMNH 116852-3 \#1-1 } \\
\text { NMNH 116852-3 \#1-2 } \\
\text { NMNH 116852-3 \#1-3 } \\
\text { NMNH 116852-3 (averag }\end{array}$ & $\begin{array}{l}\text { Basalt } \\
\text { Basalt } \\
\text { Basalt } \\
\text { ge) }\end{array}$ & & $\begin{array}{l}\text { Routine }^{\mathrm{d}} \\
\text { Long }^{\mathrm{d}} \\
\text { UTEVA }^{\mathrm{d}}\end{array}$ & $\begin{array}{l}5.6 \\
7.6 \\
7.7\end{array}$ & $\begin{array}{l}0.061 \\
0.062 \\
0.052 \\
0.058\end{array}$ & $\begin{array}{l}0.027 \\
0.027 \\
0.027 \\
0.016\end{array}$ & $\begin{array}{l}0.096 \\
0.088 \\
0.094 \\
0.093\end{array}$ & $\begin{array}{l}0.042 \\
0.042 \\
0.042 \\
0.024\end{array}$ & $\begin{array}{l}9 \\
9 \\
9\end{array}$ \\
\hline $\begin{array}{l}\text { NMNH 116852-11 \#1-1 } \\
\text { NMNH 116852-11 \#1-2 } \\
\text { NMNH 116852-11 \#1-3 } \\
\text { NMNH 116852-11 (avera }\end{array}$ & $\begin{array}{l}\text { Basalt } \\
\text { Basalt } \\
\text { Basalt } \\
\text { age) }\end{array}$ & & $\begin{array}{l}\text { Routine }^{\mathrm{d}} \\
\text { Long }^{\mathrm{d}} \\
\text { UTEVA }^{\mathrm{d}}\end{array}$ & $\begin{array}{l}5.5 \\
7.1 \\
7.3\end{array}$ & $\begin{array}{l}0.134 \\
0.133 \\
0.121 \\
0.129\end{array}$ & $\begin{array}{l}0.027 \\
0.027 \\
0.027 \\
0.016\end{array}$ & $\begin{array}{l}0.190 \\
0.200 \\
0.169 \\
0.186\end{array}$ & $\begin{array}{l}0.035 \\
0.035 \\
0.035 \\
0.020\end{array}$ & $\begin{array}{l}9 \\
9 \\
9\end{array}$ \\
\hline $\begin{array}{l}\text { GSP-1 \#1 } \\
\text { GSP-1 \#2 } \\
\text { GSP-1 \#3 } \\
\text { GSP-1 (average) }\end{array}$ & $\begin{array}{l}\text { Granodiorite } \\
\text { Granodiorite } \\
\text { Granodiorite }\end{array}$ & & $\begin{array}{l}\text { Routine }^{\mathrm{b}} \\
\text { Routine }^{\mathrm{b}} \\
\text { Routine }^{\mathrm{c}}\end{array}$ & & $\begin{array}{l}0.126 \\
0.141 \\
0.156 \\
0.143\end{array}$ & $\begin{array}{l}0.038 \\
0.046 \\
0.031 \\
0.021\end{array}$ & $\begin{array}{l}0.174 \\
0.204 \\
0.170 \\
0.178\end{array}$ & $\begin{array}{l}0.057 \\
0.066 \\
0.040 \\
0.029\end{array}$ & $\begin{array}{r}9 \\
9 \\
10\end{array}$ \\
\hline $\begin{array}{l}\text { AC-E \#1-1 } \\
\text { AC-E \#1-2 } \\
\text { AC-E \#1-3 } \\
\text { AC-E (average) }\end{array}$ & $\begin{array}{l}\text { Granite } \\
\text { Granite } \\
\text { Granite }\end{array}$ & & $\begin{array}{l}\text { Routine }^{\mathrm{d}} \\
\text { Long }^{\mathrm{d}} \\
\text { UTEVA }^{\mathrm{d}}\end{array}$ & $\begin{array}{l}1.2 \\
1.6 \\
1.7\end{array}$ & $\begin{array}{l}0.314 \\
0.330 \\
0.298 \\
0.313\end{array}$ & $\begin{array}{l}0.030 \\
0.033 \\
0.030 \\
0.018\end{array}$ & $\begin{array}{l}0.452 \\
0.480 \\
0.452 \\
0.457\end{array}$ & $\begin{array}{l}0.044 \\
0.063 \\
0.044 \\
0.028\end{array}$ & $\begin{array}{l}9 \\
9 \\
9\end{array}$ \\
\hline MAC88105 & Lunar meteorite & Find $(\mathrm{A} / \mathrm{Be})^{\mathrm{i}}$ & Routine $^{\mathrm{b}}$ & $3.4^{\mathrm{h}}$ & 0.086 & 0.034 & 0.132 & 0.049 & 8 \\
\hline Martian meteorites & & & & & & & & & \\
\hline ALHA77005 & Shergottite & Find $(A)^{i}$ & Routine $^{\mathrm{b}}$ & $15.6^{\mathrm{h}}$ & -0.008 & 0.033 & -0.026 & 0.058 & 9 \\
\hline EETA79001 & Shergottite & Find $(\mathrm{A} / \mathrm{Ae})^{\mathrm{i}}$ & Routine $^{\mathrm{b}}$ & $14.3^{\mathrm{h}}$ & -0.003 & 0.038 & -0.038 & 0.075 & 8 \\
\hline Los Angeles & Shergottite & Find & Routine $^{\mathrm{b}}$ & $16.6^{\mathrm{h}}$ & 0.008 & 0.032 & 0.010 & 0.046 & 9 \\
\hline NWA1669 & Shergottite & Find & Routine $^{\mathrm{b}}$ & & -0.016 & 0.032 & -0.030 & 0.046 & 9 \\
\hline NWA1950 & Shergottite & Find & Routine $^{\mathrm{b}}$ & $16.8^{\mathrm{h}}$ & 0.010 & 0.032 & 0.023 & 0.046 & 9 \\
\hline NWA5029 & Shergottite & Find & Routine $^{\mathrm{b}}$ & & 0.016 & 0.032 & 0.012 & 0.046 & 9 \\
\hline SaU008 & Shergottite & Find & Routine $^{\mathrm{b}}$ & & -0.023 & 0.038 & -0.042 & 0.075 & 8 \\
\hline Zagami & $\begin{array}{l}\text { Shergottite } \\
\text { Wt. Ave. of Shergottite }\end{array}$ & Fall & Routine $^{\mathrm{b}}$ & 16.0 & $\begin{array}{l}0.016 \\
0.001\end{array}$ & $\begin{array}{l}0.031 \\
0.012\end{array}$ & $\begin{array}{l}0.064 \\
0.002\end{array}$ & $\begin{array}{l}0.055 \\
0.019\end{array}$ & 9 \\
\hline Mil03346 & Nakhlite & Find $(B)^{i}$ & Routine $^{b}$ & $14.8^{\mathrm{h}}$ & 0.025 & 0.032 & 0.027 & 0.046 & 9 \\
\hline Nakhla & $\begin{array}{l}\text { Nakhlite } \\
\text { Wt. Ave. of Nakhlite }\end{array}$ & Fall & Routine $^{\text {b }}$ & $16.0^{\mathrm{h}}$ & $\begin{array}{l}0.031 \\
0.028\end{array}$ & $\begin{array}{l}0.032 \\
0.023\end{array}$ & $\begin{array}{l}0.033 \\
0.030\end{array}$ & $\begin{array}{l}0.046 \\
0.033\end{array}$ & 9 \\
\hline ALH84001 & $\begin{array}{l}\text { Orthopyroxenite } \\
W t . \text { Ave. of Mars }\end{array}$ & Find $(A / B)^{i}$ & Routine $^{\mathrm{b}}$ & $13.6^{\mathrm{h}}$ & $\begin{array}{r}-0.033 \\
0.003\end{array}$ & $\begin{array}{l}0.032 \\
0.010\end{array}$ & $\begin{array}{r}-0.034 \\
0.004\end{array}$ & $\begin{array}{l}0.046 \\
0.015\end{array}$ & 9 \\
\hline$H E D$ & & & & & & & & & \\
\hline ALHA77256 & Diogenite & Find $(A / B)^{i}$ & Routine $^{c}$ & $14.2^{\mathrm{h}}$ & -0.008 & 0.034 & 0.013 & 0.049 & 6 \\
\hline GRO95555 & Diogenite & Find $(\mathrm{A} / \mathrm{B})^{\mathrm{i}}$ & Routine $^{\mathrm{b}}$ & $12.7^{\mathrm{h}}$ & 0.019 & 0.034 & 0.042 & 0.049 & \\
\hline MET00424 & Diogenite & Find $(B)^{i}$ & Routine $^{c}$ & $15.8^{\mathrm{h}}$ & -0.034 & 0.034 & -0.074 & 0.049 & 6 \\
\hline MET00436 & Diogenite & Find $(B / C)^{i}$ & Routine $^{c}$ & $16.5^{\mathrm{h}}$ & -0.032 & 0.032 & -0.077 & 0.045 & 8 \\
\hline
\end{tabular}


Table 1 (continued)

\begin{tabular}{|c|c|c|c|c|c|c|c|c|c|}
\hline Sample & Type & $\begin{array}{l}\text { Fall/find } \\
\text { (weathering) }\end{array}$ & Chemistry & $\begin{array}{l}\mathrm{Fe} \\
\text { wt. } \%\end{array}$ & $\delta^{56} \mathrm{Fe}$ & $\begin{array}{l}95 \% \\
\text { c.i. }^{\mathrm{a}}\end{array}$ & $\delta^{57} \mathrm{Fe}$ & $\begin{array}{l}95 \% \\
\text { c. }^{\mathrm{a}}{ }^{\mathrm{a}}\end{array}$ & $n$ \\
\hline Mil07001 & Diogenite & Find $(\mathrm{A} / \mathrm{B})^{\mathrm{i}}$ & Routine $^{\mathrm{c}}$ & & -0.017 & 0.032 & -0.036 & 0.045 & 8 \\
\hline NWA1461 & Diogenite & Find & Routine $^{\mathrm{c}}$ & $7.5^{\mathrm{h}}$ & 0.027 & 0.038 & 0.009 & 0.057 & 4 \\
\hline NWA5480 & Diogenite & Find & Routine $^{\mathrm{b}}$ & & 0.015 & 0.034 & 0.040 & 0.049 & 8 \\
\hline Shalka & Diogenite & Fall & Routine $^{\mathrm{c}}$ & $12.7^{\mathrm{h}}$ & -0.011 & 0.038 & -0.034 & 0.057 & 4 \\
\hline \multirow[t]{2}{*}{ Tatahouine } & Diogenite & Fall & Routine $^{\mathrm{b}}$ & 9.0 & 0.013 & 0.030 & 0.036 & 0.049 & 9 \\
\hline & Wt. Ave. of Diogenite & & & & -0.004 & 0.011 & -0.011 & 0.016 & \\
\hline Frankfort & Howardite & Fall & Routine $^{\mathrm{b}}$ & 12.4 & -0.002 & 0.038 & -0.015 & 0.057 & 9 \\
\hline Kapoeta & Howardite & Fall & Routine $^{\mathrm{b}}$ & 8.7 & 0.026 & 0.030 & 0.042 & 0.049 & 9 \\
\hline \multirow[t]{2}{*}{ Petersburg } & Howardite/polymict eucrite & Fall & Routine $^{\mathrm{b}}$ & 8.3 & 0.035 & 0.038 & 0.056 & 0.057 & 9 \\
\hline & Wt. Ave. of Howardite & & & & 0.021 & 0.020 & 0.029 & 0.031 & \\
\hline NWA1240 & Eucrite (Ungrouped) & Find & Routine $^{\mathrm{d}}$ & $15.7^{\mathrm{h}}$ & 0.016 & 0.031 & -0.026 & 0.043 & 6 \\
\hline Pasamonte & Eucrite (Ungrouped) & Fall & Routine $^{\mathrm{b}}$ & 11.5 & 0.019 & 0.030 & 0.063 & 0.045 & 8 \\
\hline EET87548 \#1 & Eucrite (Cumulate) & Find $(B / C)^{i}$ & Routine $^{\mathrm{d}}$ & 15.1 & -0.023 & 0.034 & -0.029 & 0.051 & 9 \\
\hline EET87548 \#2 & Eucrite (Cumulate) & Find $(B / C)^{i}$ & Routine $^{\mathrm{b}}$ & 15.4 & -0.017 & 0.034 & -0.030 & 0.049 & 8 \\
\hline EET87548 \#3 & Eucrite (Cumulate) & Find $(B / C)^{i}$ & Routine $^{d}$ & & -0.014 & 0.030 & -0.024 & 0.059 & 9 \\
\hline EET87548 (average) & & & & & -0.018 & 0.019 & -0.028 & 0.030 & \\
\hline Moore County \#1 & Eucrite (Cumulate) & Fall & Routine $^{\mathrm{b}}$ & & -0.004 & 0.038 & -0.008 & 0.057 & 9 \\
\hline Moore County \#2 & Eucrite (Cumulate) & Fall & Routine $^{b}$ & 7.4 & 0.010 & 0.030 & 0.040 & 0.049 & 9 \\
\hline Moore County (average) & & & & & 0.005 & 0.024 & 0.020 & 0.037 & \\
\hline Serra de Magé \#1 & Eucrite (Cumulate) & Fall & Routine $^{\mathrm{d}}$ & 12.3 & 0.001 & 0.034 & 0.026 & 0.051 & 9 \\
\hline Serra de Magé \#2-1 & Eucrite (Cumulate) & Fall & Routine $^{\mathrm{d}}$ & 12.0 & 0.017 & 0.028 & 0.025 & 0.039 & 9 \\
\hline Serra de Magé \#2-2 & Eucrite (Cumulate) & Fall & Long $^{d}$ & 16.1 & 0.019 & 0.028 & 0.032 & 0.039 & 9 \\
\hline Serra de Magé \#2-3 & Eucrite (Cumulate) & Fall & UTEVA $^{\mathrm{d}}$ & 16.6 & 0.006 & 0.028 & 0.009 & 0.039 & 9 \\
\hline \multirow[t]{2}{*}{ Serra de Magé (average) } & & & & & 0.012 & 0.015 & 0.023 & 0.021 & \\
\hline & $\begin{array}{l}\text { Wt. Ave. of Eucrite } \\
\text { (Cumulate) }\end{array}$ & & & & 0.001 & 0.010 & 0.009 & 0.015 & \\
\hline Cachari & Eucrite $(\mathrm{MG})^{\mathrm{e}}$ & Find & Routine $^{\mathrm{b}}$ & $15.9^{\mathrm{h}}$ & 0.002 & 0.030 & 0.011 & 0.045 & 8 \\
\hline Camel Donga & Eucrite $(\mathrm{MG})^{\mathrm{e}}$ & Find & Routine $^{\mathrm{d}}$ & 15.3 & 0.019 & 0.034 & 0.034 & 0.051 & 9 \\
\hline Jonzac \#1 & Eucrite $(\mathrm{MG})^{\mathrm{e}}$ & Fall & Routine $^{\mathrm{b}}$ & 15.0 & 0.025 & 0.035 & 0.031 & 0.057 & 8 \\
\hline Jonzac \#2 & Eucrite $(\mathrm{MG})^{\mathrm{e}}$ & Fall & Routine $^{b}$ & 11.5 & 0.007 & 0.034 & 0.051 & 0.049 & 8 \\
\hline Jonzac \#3-1 & Eucrite $(\mathrm{MG})^{\mathrm{e}}$ & Fall & Routine $^{\mathrm{d}}$ & 9.8 & 0.008 & 0.032 & 0.028 & 0.045 & 9 \\
\hline Jonzac \#3-2 & Eucrite $(\mathrm{MG})^{\mathrm{e}}$ & Fall & Long $^{\mathrm{d}}$ & 14.8 & -0.006 & 0.032 & -0.007 & 0.045 & 9 \\
\hline Jonzac \#3-3 & Eucrite $(\mathrm{MG})^{\mathrm{e}}$ & Fall & UTEVA $^{\mathrm{d}}$ & 14.6 & 0.010 & 0.032 & 0.035 & 0.045 & 9 \\
\hline Jonzac (average) & & & & & 0.008 & 0.015 & 0.026 & 0.021 & \\
\hline Juvinas \#1 & Eucrite $(\mathrm{MG})^{\mathrm{e}}$ & Fall & Routine $^{\mathrm{b}}$ & & 0.015 & 0.038 & 0.035 & 0.057 & 9 \\
\hline Juvinas \#2-1 & Eucrite $(\mathrm{MG})^{\mathrm{e}}$ & Fall & Routine $^{\mathrm{d}}$ & 9.9 & -0.025 & 0.028 & -0.019 & 0.040 & 9 \\
\hline Juvinas \#2-2 & Eucrite $(\mathrm{MG})^{\mathrm{e}}$ & Fall & Long $^{\mathrm{d}}$ & 14.0 & -0.022 & 0.028 & -0.024 & 0.040 & 9 \\
\hline Juvinas \#2-3 & Eucrite $(\mathrm{MG})^{\mathrm{e}}$ & Fall & UTEVA $^{\mathrm{d}}$ & 13.6 & -0.017 & 0.028 & -0.021 & 0.040 & 9 \\
\hline Juvinas \#3-1 & Eucrite $(\mathrm{MG})^{\mathrm{e}}$ & Fall & Routine $^{\mathrm{d}}$ & 9.9 & -0.026 & 0.035 & -0.038 & 0.050 & 9 \\
\hline Juvinas \#3-2 & Eucrite $(\mathrm{MG})^{\mathrm{e}}$ & Fall & Long $^{\mathrm{d}}$ & 14.0 & -0.023 & 0.035 & -0.036 & 0.050 & 9 \\
\hline Juvinas \#3-3 & Eucrite $(\mathrm{MG})^{\mathrm{e}}$ & Fall & UTEVA $^{\mathrm{d}}$ & 13.6 & -0.037 & 0.035 & -0.059 & 0.050 & 9 \\
\hline Juvinas (average) & & & & & -0.020 & 0.012 & -0.024 & 0.017 & \\
\hline \multirow[t]{2}{*}{ NWA049 } & Eucrite $(\mathrm{MG})^{\mathrm{e}}$ & Find & Routine $^{\mathrm{b}}$ & $15.4^{\mathrm{h}}$ & 0.023 & 0.029 & 0.033 & 0.047 & 9 \\
\hline & Wt. Ave. of Eucrite $(M G)^{\mathrm{e}}$ & & & & -0.004 & 0.008 & 0.001 & 0.012 & \\
\hline Agoult & Eucrite (Residual) & Find & Routine $^{\mathrm{b}}$ & $15.2^{\mathrm{h}}$ & 0.033 & 0.034 & 0.039 & 0.049 & 8 \\
\hline \multirow[t]{2}{*}{ Dag945 } & Eucrite (Residual) & Find $(\mathrm{W} 1)^{\mathrm{i}}$ & Routine $^{\mathrm{c}}$ & $15.6^{\mathrm{h}}$ & 0.028 & 0.036 & 0.037 & 0.052 & 5 \\
\hline & $\begin{array}{l}\text { Wt. Ave. of Eucrite } \\
\text { (Residual) }\end{array}$ & & & & 0.031 & 0.025 & 0.038 & 0.036 & \\
\hline Nuevo Laredo & Eucrite $(\mathrm{NL})^{\mathrm{f}}$ & Find & Routine $^{\mathrm{c}}$ & $16.2^{\mathrm{h}}$ & 0.031 & 0.036 & 0.056 & 0.052 & 5 \\
\hline \multirow[t]{2}{*}{ Sahara02501 } & Eucrite $(\mathrm{NL})^{\mathrm{f}}$ & Find & Routine $^{\mathrm{c}}$ & 16.3 & 0.037 & 0.029 & 0.053 & 0.049 & 8 \\
\hline & Wt. Ave. of Eucrite $(N L)^{\mathrm{f}}$ & & & & 0.035 & 0.023 & 0.054 & 0.036 & \\
\hline Bouvante \#1 & Eucrite $(\mathrm{ST})^{\mathrm{g}}$ & Find & Routine $^{\mathrm{d}}$ & 15.1 & 0.049 & 0.034 & 0.096 & 0.051 & 9 \\
\hline Bouvante \#2-1 & Eucrite $(\mathrm{ST})^{\mathrm{g}}$ & Find & Routine $^{\mathrm{d}}$ & 10.7 & 0.032 & 0.028 & 0.036 & 0.036 & 9 \\
\hline Bouvante \#2-2 & Eucrite $(\mathrm{ST})^{\mathrm{g}}$ & Find & Long $^{\mathrm{d}}$ & 16.6 & 0.038 & 0.028 & 0.054 & 0.036 & 9 \\
\hline Bouvante \#2-3 & Eucrite $(\mathrm{ST})^{\mathrm{g}}$ & Find & UTEVA $^{\mathrm{d}}$ & 15.9 & 0.026 & 0.028 & 0.054 & 0.036 & 9 \\
\hline Bouvante (average) & & & & & 0.035 & 0.015 & 0.055 & 0.019 & \\
\hline NWA2061 & Eucrite $(\mathrm{ST})^{\mathrm{g}}$ & Find & Routine $^{\mathrm{b}}$ & 14.4 & 0.018 & 0.029 & 0.031 & 0.047 & 9 \\
\hline NWA4523 \#1 & Eucrite $(\mathrm{ST})^{\mathrm{g}}$ & Find & Routine $^{\mathrm{b}}$ & $13.3^{\mathrm{h}}$ & 0.077 & 0.034 & 0.118 & 0.049 & 8 \\
\hline NWA4523 \#2 & Eucrite $(\mathrm{ST})^{\mathrm{g}}$ & Find & Routine $^{\mathrm{d}}$ & & 0.071 & 0.034 & 0.097 & 0.051 & 9 \\
\hline
\end{tabular}


Table 1 (continued)

\begin{tabular}{|c|c|c|c|c|c|c|c|c|c|}
\hline Sample & Type & $\begin{array}{l}\text { Fall/find } \\
\text { (weathering) }\end{array}$ & Chemistry & $\begin{array}{l}\mathrm{Fe} \\
\text { wt. } \%\end{array}$ & $\delta^{56} \mathrm{Fe}$ & $\begin{array}{l}95 \% \\
\text { c.i. }^{a} \\
\end{array}$ & $\delta^{57} \mathrm{Fe}$ & $\begin{array}{l}95 \% \\
\text { c.i. }^{a}\end{array}$ & $n$ \\
\hline $\begin{array}{l}\text { NWA4523 \#3-1 } \\
\text { NWA4523 \#3-2 } \\
\text { NWA4523 \#3-3 } \\
\text { NWA4523 (average) }\end{array}$ & $\begin{array}{l}\text { Eucrite }(\mathrm{ST})^{\mathrm{g}} \\
\text { Eucrite }(\mathrm{ST})^{\mathrm{g}} \\
\text { Eucrite }(\mathrm{ST})^{\mathrm{g}}\end{array}$ & $\begin{array}{l}\text { Find } \\
\text { Find } \\
\text { Find }\end{array}$ & $\begin{array}{l}\text { Routine }^{\mathrm{d}} \\
\text { Long }^{\mathrm{d}} \\
\text { UTEVA }^{\mathrm{d}}\end{array}$ & & $\begin{array}{l}0.056 \\
0.052 \\
0.060 \\
0.061\end{array}$ & $\begin{array}{l}0.027 \\
0.027 \\
0.027 \\
0.013\end{array}$ & $\begin{array}{l}0.071 \\
0.095 \\
0.095 \\
0.093\end{array}$ & $\begin{array}{l}0.039 \\
0.039 \\
0.039 \\
0.019\end{array}$ & $\begin{array}{l}9 \\
9 \\
9\end{array}$ \\
\hline $\begin{array}{l}\text { Pomozdino } \\
\text { Stannern \#1 } \\
\text { Stannern \#2 } \\
\text { Stannern \#3 } \\
\text { Stannern \#4 } \\
\text { Stannern \#5-1 } \\
\text { Stannern \#5-2 } \\
\text { Stannern \#5-3 } \\
\text { Stannern (average) }\end{array}$ & $\begin{array}{l}\text { Eucrite }(\mathrm{ST})^{\mathrm{g}} \\
\text { Eucrite }(\mathrm{ST})^{\mathrm{g}} \\
\text { Eucrite }(\mathrm{ST})^{\mathrm{g}} \\
\text { Eucrite }(\mathrm{ST})^{\mathrm{g}} \\
\text { Eucrite }(\mathrm{ST})^{\mathrm{g}} \\
\text { Eucrite }(\mathrm{ST})^{\mathrm{g}} \\
\text { Eucrite }(\mathrm{ST})^{\mathrm{g}} \\
\text { Eucrite }(\mathrm{ST})^{\mathrm{g}}\end{array}$ & $\begin{array}{l}\text { Find } \\
\text { Fall } \\
\text { Fall } \\
\text { Fall } \\
\text { Fall } \\
\text { Fall } \\
\text { Fall } \\
\text { Fall }\end{array}$ & $\begin{array}{l}\text { Routine }^{\mathrm{d}} \\
\text { Routine }^{\mathrm{b}} \\
\text { Routine }^{\mathrm{d}} \\
\text { Routine }^{\mathrm{d}} \\
\text { Routine }^{\mathrm{d}} \\
\text { Routine }^{\mathrm{d}} \\
\text { Longd }^{\mathrm{d}} \\
\text { UTEVA }^{\mathrm{d}}\end{array}$ & $\begin{array}{l}15.2 \\
14.2 \\
13.7 \\
\\
14.5 \\
12.0 \\
12.5 \\
15.6\end{array}$ & $\begin{array}{l}0.034 \\
0.072 \\
0.036 \\
0.049 \\
0.053 \\
0.047 \\
0.035 \\
0.037 \\
0.045\end{array}$ & $\begin{array}{l}0.034 \\
0.035 \\
0.045 \\
0.045 \\
0.045 \\
0.026 \\
0.026 \\
0.026 \\
0.012\end{array}$ & $\begin{array}{l}0.060 \\
0.095 \\
0.079 \\
0.102 \\
0.071 \\
0.078 \\
0.073 \\
0.059 \\
0.074\end{array}$ & $\begin{array}{l}0.051 \\
0.057 \\
0.065 \\
0.065 \\
0.065 \\
0.032 \\
0.032 \\
0.032 \\
0.016\end{array}$ & $\begin{array}{l}9 \\
8 \\
9 \\
9 \\
9 \\
9 \\
9 \\
9\end{array}$ \\
\hline Yamato75011 & $\begin{array}{l}\text { Eucrite }(\mathrm{ST})^{\mathrm{g}} \\
\text { Wt. Ave. of Eucrite }(\mathrm{ST})^{\mathrm{g}}\end{array}$ & Find $(A)^{\mathrm{i}}$ & Routine $^{\mathrm{b}}$ & 15.2 & $\begin{array}{l}0.043 \\
0.045\end{array}$ & $\begin{array}{l}0.029 \\
0.007\end{array}$ & $\begin{array}{l}0.092 \\
0.073\end{array}$ & $\begin{array}{l}0.047 \\
0.010\end{array}$ & 9 \\
\hline $\begin{array}{l}\text { Angrite } \\
\text { D'Orbigny } \\
\text { LEW86010 } \\
\text { NWA1670 \#1 } \\
\text { NWA1670 \#2 } \\
\text { NWA1670 \#3-1 } \\
\text { NWA1670 \#3-2 } \\
\text { NWA1670 \#3-3 } \\
\text { NWA1670 (average) }\end{array}$ & $\begin{array}{l}\text { Angrite } \\
\text { Angrite } \\
\text { Angrite } \\
\text { Angrite } \\
\text { Angrite } \\
\text { Angrite } \\
\text { Angrite }\end{array}$ & $\begin{array}{l}\text { Find } \\
\text { Find }(\mathrm{A} / \mathrm{B})^{\mathrm{i}} \\
\text { Find } \\
\text { Find } \\
\text { Find } \\
\text { Find } \\
\text { Find }\end{array}$ & $\begin{array}{l}\text { Routine }^{\mathrm{d}} \\
\text { Routine }^{\mathrm{b}} \\
\text { Routine }^{\mathrm{b}} \\
\text { Routine }^{\mathrm{d}} \\
\text { Routine }^{\mathrm{d}} \\
\text { Long }^{\mathrm{d}} \\
\text { UTEVA }^{\mathrm{d}}\end{array}$ & $\begin{array}{l}19.2^{\mathrm{h}} \\
14.2^{\mathrm{h}} \\
14.4^{\mathrm{h}}\end{array}$ & $\begin{array}{l}0.139 \\
0.139 \\
0.167 \\
0.125 \\
0.148 \\
0.113 \\
0.154 \\
0.142\end{array}$ & $\begin{array}{l}0.030 \\
0.043 \\
0.033 \\
0.036 \\
0.030 \\
0.030 \\
0.030 \\
0.014\end{array}$ & $\begin{array}{l}0.193 \\
0.179 \\
0.253 \\
0.210 \\
0.225 \\
0.172 \\
0.223 \\
0.212\end{array}$ & $\begin{array}{l}0.062 \\
0.059 \\
0.058 \\
0.058 \\
0.038 \\
0.038 \\
0.038 \\
0.019\end{array}$ & $\begin{array}{l}9 \\
9 \\
9 \\
9 \\
9 \\
9 \\
9\end{array}$ \\
\hline $\begin{array}{l}\text { NWA1296 } \\
\text { NWA6291 } \\
\text { NWA2999 } \\
\text { NWA4801 } \\
\text { Sahara99555 \#1 } \\
\text { Sahara99555 \#2 } \\
\text { Sahara99555 \#3 } \\
\text { Sahara99555 (average) }\end{array}$ & $\begin{array}{l}\text { Angrite } \\
\text { Angrite } \\
\text { Angrite } \\
\text { Angrite } \\
\text { Angrite } \\
\text { Angrite } \\
\text { Angrite }\end{array}$ & $\begin{array}{l}\text { Find } \\
\text { Find } \\
\text { Find } \\
\text { Find } \\
\text { Find } \\
\text { Find } \\
\text { Find }\end{array}$ & $\begin{array}{l}\text { Routine }^{\mathrm{b}} \\
\text { Routine }^{\mathrm{d}} \\
\text { Routine }^{\mathrm{d}} \\
\text { Routine }^{\mathrm{d}} \\
\text { Routine }^{\mathrm{d}} \\
\text { Routine }^{\mathrm{b}} \\
\text { Routine }^{\mathrm{d}}\end{array}$ & $\begin{array}{l}19.4^{\mathrm{h}} \\
24.2^{\mathrm{h}} \\
18.0^{\mathrm{h}}\end{array}$ & $\begin{array}{l}0.104 \\
0.052 \\
0.124 \\
0.135 \\
0.116 \\
0.107 \\
0.110 \\
0.110\end{array}$ & $\begin{array}{l}0.033 \\
0.030 \\
0.030 \\
0.030 \\
0.040 \\
0.033 \\
0.030 \\
0.019\end{array}$ & $\begin{array}{l}0.185 \\
0.116 \\
0.183 \\
0.219 \\
0.165 \\
0.136 \\
0.141 \\
0.144\end{array}$ & $\begin{array}{l}0.058 \\
0.062 \\
0.062 \\
0.062 \\
0.081 \\
0.058 \\
0.062 \\
0.038\end{array}$ & $\begin{array}{l}9 \\
9 \\
9 \\
9 \\
9 \\
9 \\
9\end{array}$ \\
\hline & Wt. Ave. of Angrite & & & & 0.123 & 0.009 & 0.192 & 0.014 & \\
\hline
\end{tabular}

${ }^{\text {a }}$ Uncertainties are $95 \%$ confidence intervals (see Dauphas et al. (2009b) for details).

b Samples chemically prepared at the Washington University in St. Louis and measured at the University of Chicago.

${ }^{c}$ Samples both chemically prepared and measured at Washington University in St. Louis.

${ }^{\mathrm{d}}$ Samples both chemically prepared and measured at the University of Chicago.

e Eucrite $(\mathrm{MG})=$ Eucrite main group.

${ }^{\mathrm{f}}$ Eucrite $(\mathrm{NL})=$ Eucrite Nuevo Laredo Trend.

g Eucrite $(\mathrm{ST})=$ Eucrite Stannern Trend.

${ }^{\mathrm{h}}$ Reference data: MAC88105 (Jarosewich, 1990); NWA1950 (Gillet et al., 2005); Los Angeles (Warren et al., 2000); EETA79001, ALHA77005, Nakhla, ALH84001 (Lodders, 1998); Mil03346 (Day et al., 2006); GRO95555, MET00436, MET00424 (Barrat et al., 2008); NWA1461 (Warren et al., 2009); Shalka (McCarthy et al., 1972); ALHA77256 (Sack et al., 1991); NWA1240, NWA049 (Barrat et al., 2003); Cachari (Barrat et al., 2000); Agoult, Dag945 (Yamaguchi et al., 2009); Nuevo Laredo, NWA4523 (Barrat et al., 2007b); D’Orbigny, Sahara99555 (Mittlefehldt et al., 2002); LEW86010 (McKay et al., 1988); NWA1670 (Jambon et al., 2008); NWA1296 (Jambon et al., 2005); NWA2999 (Gellissen et al., 2007).

i Weathering conditions are from MetBase (version 7.1). Weathering index A, B or C represents "minor", "moderate" or "severe" rustiness, respectively. Letter "e" represents evaporite minerals visible. Weathering grade W0 (fresh) to W6 (most weathered) is also shown if available.

${ }^{\mathrm{j}}$ These samples have been added $\mathrm{V}$ and $\mathrm{Cr}$ to double-check the purification of iron during chemistry.

from the Colombia River, Oregon. BIR-1 is a basalt from Iceland. BHVO-2 is a Hawaiian lava basalt. AGV-1 and 2 are andesites from the Guano Valley, Oregon. GSP-1 is a granodiorite from Silver Plume, Colorado. AC-E is a granite from Ailsa Craig Island, Scotland. In addition, three modern island arc basalts (IABs) from New Britain (NMNH 116852-1, 116852-3 and 116852-11) were also analyzed here to test the accuracy of the measurements (see Dauphas et al. (2009a) for a detailed discussion about IABs). 
Lunar highland meteorite, MAC88105, is a polymict breccia. It is dominated by ferroan anorthosite lithology and contains small low-Ti basaltic clasts (Jolliff et al., 1991; Neal et al., 1991). The bulk composition of this lunar highland meteorites is different from those of highland rocks sampled by Apollo project (Koeberl et al., 1991; Lindstrom et al., 1991) and might represent a different feldspathic highlands terrane (Warren et al., 1989). Orbital remote sensing composition data shows that lunar highland meteorites are reasonably representative of lunar surface (Korotev et al., 2003).

The martian meteorites studied include eight shergottites, two nakhlites and ALH84001. Shergottites are basaltic or lherzolitic rocks. Basaltic shergottites (e.g., Zagami, EETA79001) mainly consist of clinopyroxene and plagioclase (Mittlefehldt et al., 1998). Lherzolitic shergottites (e.g., ALHA77005) are cumulates, consisting of magnesian olivine, clinopyroxene and chromite (Mittlefehldt et al., 1998). Nakhlites are clinopyroxenites made of augite and a small amount of olivine (Mittlefehldt et al., 1998). ALH84001 is a cumulate orthopyroxenite.

The HED meteorites reported in this study include 20 eucrites, three howardites and nine diogenites. Based on spectroscopic observations, HED meteorites are generally recognized as being derived from asteroid 4-Vesta (McCord et al., 1970; Drake, 2001). Diogenites are orthopyroxenites, consisting chiefly of $\sim 90$ vol $\%$ coarse-grained orthopyroxene, and accessory minerals including olivine, chromite, troilite, and metal (Mittlefehldt et al., 1998). Eucrites are basalts (non-cumulates) or cumulate gabbros. Basaltic eucrites contain pigeonite, plagioclase and a minor amount of silica, ilmenite and chromite (Duke and Silver, 1967). Basaltic eucrites can be subdivided based on geochemical characteristics: "Main Group" (MG), "Nuevo Laredo Trend" (NL), and "Stannern Trend" (ST) (Stolper, 1977; Reid and Barnard, 1979; Yamaguchi et al., 2009). These three groups are very similar in major element compositions, however they are different in trace element abundances. The Stannern Trend shows a significant increase in incompatible trace elements without changes in $\mathrm{Mg \#}$ compared to the Main Group; while the Nuevo
Laredo Trend shows a slight increase in incompatible trace elements and a decrease in $\mathrm{Mg \#}$ compared to the Main Group. Cumulate eucrites have mineral assemblages similar to basaltic eucrites, but they display cumulate textures and their pyroxenes are Mg-rich. Howardites are polymict breccias and are mixtures primarily of diogenite and eucrite clasts. In addition, we have analyzed Pasamonte and NWA1240, two ungrouped eucrites recently found to have oxygen isotope compositions distinct from all other HEDs (Scott et al., 2009). Pasamonte is a polymict breccia that displays highly unequilibrated basaltic clasts (Takeda et al., 1978), and possibly records fluid-rock interactions (Schwartz and McCallum, 2005). NWA1240 is an unbrecciated stone, with unique features. It displays a porphyritic texture consisting of skeletal hollow low-Ca pyroxene phenocrysts set in a variolitic (fan-spherulitic) mesostasis of fine elongate pyroxene and plagioclase crystals. Pyroxenes are highly unequilibrated and their compositions range from $\mathrm{En}_{76}$ to $\mathrm{En}_{0.6}$. Although its texture indicates a rock formed from a rapidly-cooled melt, its composition is more akin to cumulate eucrites and led Barrat et al. (2003) to propose an impact-melt origin. Nevertheless, these ungrouped eucrites are probably not from asteroid 4-Vesta, but from Vesta-like asteroid parent-bodies (Scott et al., 2009).

Angrites are among the oldest basaltic rocks in the Solar System (Baker et al., 2005; Nyquist et al., 2009; Dauphas and Chaussidon, 2011). Only 19 angrites are recognized. Eight representative ones were analyzed here: D'Orbigny, NWA1296 and Sahara99555 consist of mainly Al-Ti-diopside-hedenbergite, Ca-rich olivine, anorthite and spinel olivine; they are also similar in bulk chemistry (Jambon et al., 2005); LEW 86010 consists of $\sim 58$ vol \% Al-Ti-diopsidehedenbergite, $21 \mathrm{vol} \%$ plagioclase, $20 \mathrm{vol} \%$ olivine and minor amount of spinel, troilite, and $\mathrm{Fe}-\mathrm{Ni}$ metal (Prinz et al., 1988); NWA1670 consists of large olivine xenocrysts, and fine-grained groundmass with pyroxene, anorthite and olivine (Jambon et al., 2008); NWA4801 is composed of Al-Ti-diopside-hedenbergite and pure anorthite (Irving and Kuehner, 2007); NWA2999 contains 64 vol \% Ca-rich olivine, 23 vol \% Al-Ti-diopside-hedenbergite, 4 vol \% spinel, 1 vol \% plagioclase and 8 vol \% metal (Kuehner et al.,

Table 2

Comparison of the three iron purification chromatography protocols used in this study (see text for references).

\begin{tabular}{|c|c|c|c|}
\hline & Routine column & Long column & UTEVA column \\
\hline$\overline{\text { Column }}$ & $\begin{array}{l}\text { Disposable Bio-rad poly prep } \\
\text { polyethylene column ( } 9 \mathrm{~cm} \text { length) }\end{array}$ & $\begin{array}{l}\text { Reusable Savillex PFA column } \\
(10.5 \mathrm{~cm} \text { length, } 0.62 \mathrm{~cm} \text { diameter })\end{array}$ & $\begin{array}{l}\text { Pre-packaged Eichrom } \\
\text { U/Teva cartridges }\end{array}$ \\
\hline Resin & $\begin{array}{l}1 \mathrm{~mL} \\
\text { AG1-X8 200-400 mesh anion-exchange } \\
\text { resin }\end{array}$ & $\begin{array}{l}3 \mathrm{~mL} \\
\text { AG1-X8 200-400 mesh anion- } \\
\text { exchange } \\
\text { resin }\end{array}$ & $\begin{array}{l}1 \mathrm{~mL} \\
\text { Eichrom U/Teva resin }\end{array}$ \\
\hline Conditioning & $10 \mathrm{~mL} 6 \mathrm{M} \mathrm{HCl}$ & $4 \mathrm{~mL} 10 \mathrm{M} \mathrm{HCl}$ & $6 \mathrm{~mL} 4 \mathrm{M} \mathrm{HCl}$ \\
\hline Matrix elution & $\begin{array}{l}8 \mathrm{~mL} 6 \mathrm{M} \mathrm{HCl} \\
(0.5+0.5+1+2+4 \mathrm{~mL} \text { increments })\end{array}$ & $\begin{array}{l}5 \mathrm{~mL} 10 \mathrm{M} \mathrm{HCl} \\
(0.5+0.5+1+3 \mathrm{~mL} \text { increments }) \\
22 \mathrm{~mL} 4 \mathrm{M} \mathrm{HCl} \\
(0.5+0.5+1+5+5+10 \mathrm{~mL} \\
\text { increments })\end{array}$ & $\begin{array}{l}25 \mathrm{~mL} 4 \mathrm{M} \mathrm{HCl} \\
(0.5+0.5+1+5+8+10 \mathrm{~mL} \\
\text { increments })\end{array}$ \\
\hline $\begin{array}{l}\text { Iron } \\
\text { collecting }\end{array}$ & $\begin{array}{l}9 \mathrm{~mL} 0.4 \mathrm{M} \mathrm{HCl} \\
(0.5+0.5+1+3+4 \mathrm{~mL} \text { increments })\end{array}$ & $\begin{array}{l}8 \mathrm{~mL} 0.4 \mathrm{M} \mathrm{HCl} \\
(0.5+0.5+1+2+4 \mathrm{~mL} \text { increments })\end{array}$ & $\begin{array}{l}12 \mathrm{~mL} 0.4 \mathrm{M} \mathrm{HCl} \\
(0.5+0.5+1+5+5 \mathrm{~mL} \text { increments })\end{array}$ \\
\hline
\end{tabular}



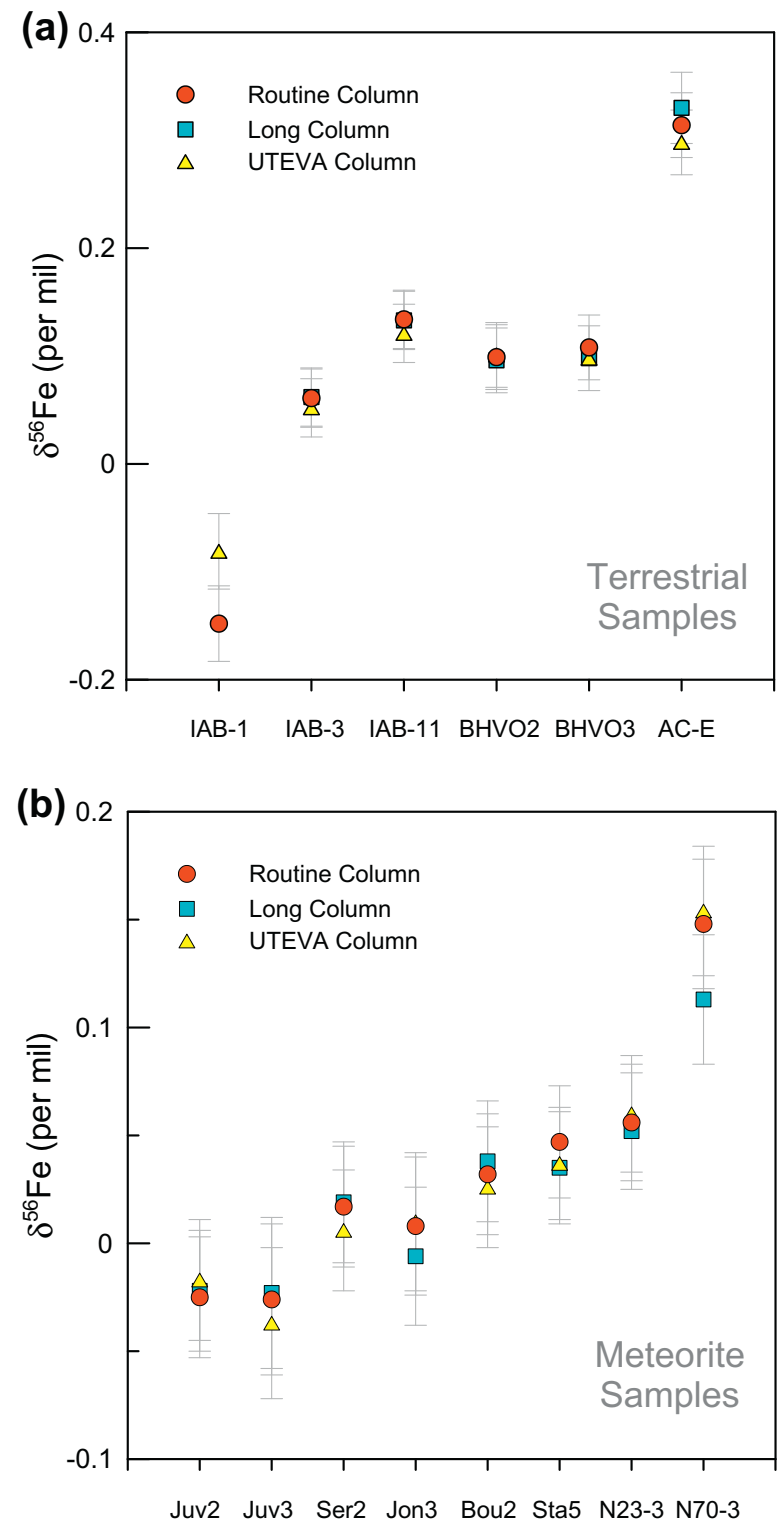

Fig. 1. Iron isotope results of the samples that were purified independently by three different purification chemistries, namely "Routine Columns", "Long Columns" and "UTEVA Columns". For a detailed description of these purification chemistry protocols, please see Table 2 and text. Top panel: terrestrial samples. IAB$1=\mathrm{NMNH} \quad 116852-1, \quad$ IAB-3 = NMNH $116852-3, \quad$ IAB-11 = NMNH 116852-11, BHVO2 = BHVO-2 \#2, BHVO3 = BHVO-2 \#3, AC-E = AC-E \#1. Bottom panel: meteorite samples. Juv2= Juvinas \#2, Juv3 = Juvinas \#3, Ser2 = Serra de Magé \#2, Jon3 = Jonzac \#3, Bou $2=$ Bouvante \#2, Sta5 = Stannern \#5, N23$3=$ NWA4523 \#3, N70-3 = NWA1670 \#3.

2006); NWA6291 is the most recently found angrite and is possibly paired with NWA2999 (Bouvier et al., 2011).

\subsection{Analytical methods}

Several hundred milligrams of meteorite was crushed in an agate mortar to ensure representative sampling. Be-

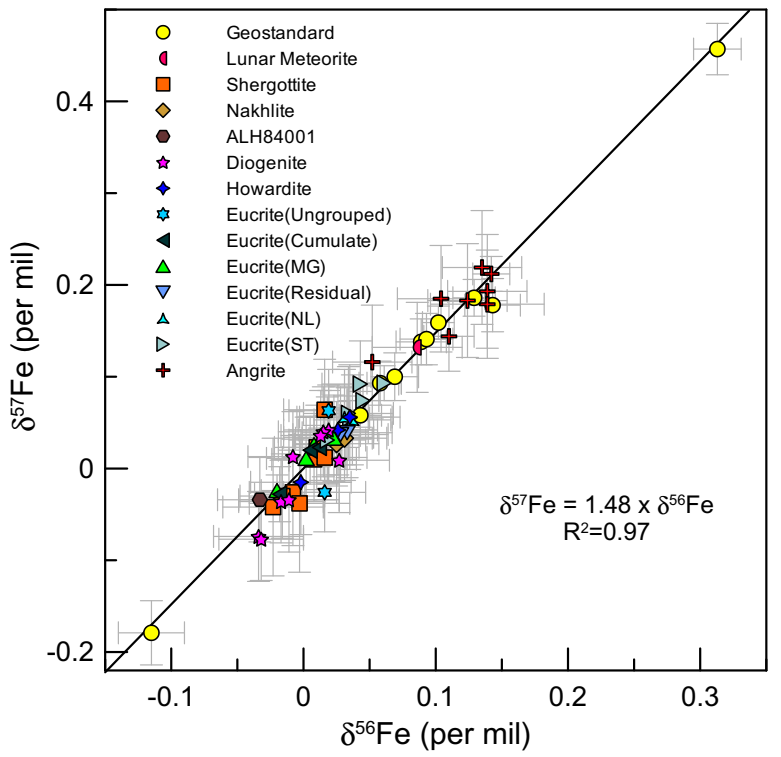

Fig. 2. Mass-dependent isotope fractionation of all samples in this study, shown in a $\delta^{56} \mathrm{Fe}-\delta^{57} \mathrm{Fe}$ space. Eucrite $(\mathrm{MG})=$ Eucrite Main Group, Eucrite $(\mathrm{NL})=$ Eucrite Nuevo Laredo Trend, Eucrite (ST) = Eucrite Stannern Trend. Each point represents one meteorite/geostandard. For replicate measurements in Table 1, only the average is plotted.

tween 5 and $10 \mathrm{mg}$ of crushed material was fully digested by sequential mixtures of $\mathrm{HF}-\mathrm{HNO}_{3}$ and $\mathrm{HNO}_{3}-\mathrm{HCl}$. Iron in the dissolved samples was then purified using anionexchange chromatography (see Table 2 for the detailed procedure; Strelow, 1980; Dauphas et al., 2004, 2009b). The purification was repeated twice, thus ensuring the removal of all the matrix elements. Most separation chemistries were carried out in the clean laboratory at Washington University in St. Louis (WUSTL) and supplemented by chemistries for selected samples at the University of Chicago (UofC) to confirm inter-laboratory concordance and reproducibility. Most iron isotope analyses were performed with a Thermo Scientific Neptune MC-ICP-MS at the UofC and some were done with a Thermo Scientific Neptune Plus MC-ICP-MS at WUSTL, both following identical protocol described in Dauphas et al. (2009b).

In order to further confirm the validity of the iron separation technique used in this study (marked as "routine" chemistry), two other Fe separation techniques were applied to selected samples (marked as "long" and "UTEVA" chemistry in Table 2). The "long" column method uses long Teflon columns $(10.5 \mathrm{~cm})$ filled with $3 \mathrm{ml}$ AG1-X8 anionexchange resin (Dauphas et al., 2009b; Craddock and Dauphas, 2011). The "UTEVA" column method uses prepackaged $1 \mathrm{~mL}$ Eichrom UTEVA resin cartridges (Horwitz et al., 1992; Tissot and Dauphas, 2011). All samples measured following purification by the three chemistry protocols yield identical iron isotope compositions within analytical uncertainty (Fig. 1 and Table 1 ).

All data are reported in $\delta^{56} \mathrm{Fe}$ and $\delta^{57} \mathrm{Fe}$ notations relative to the isotopic reference IRMM-014, defined as 


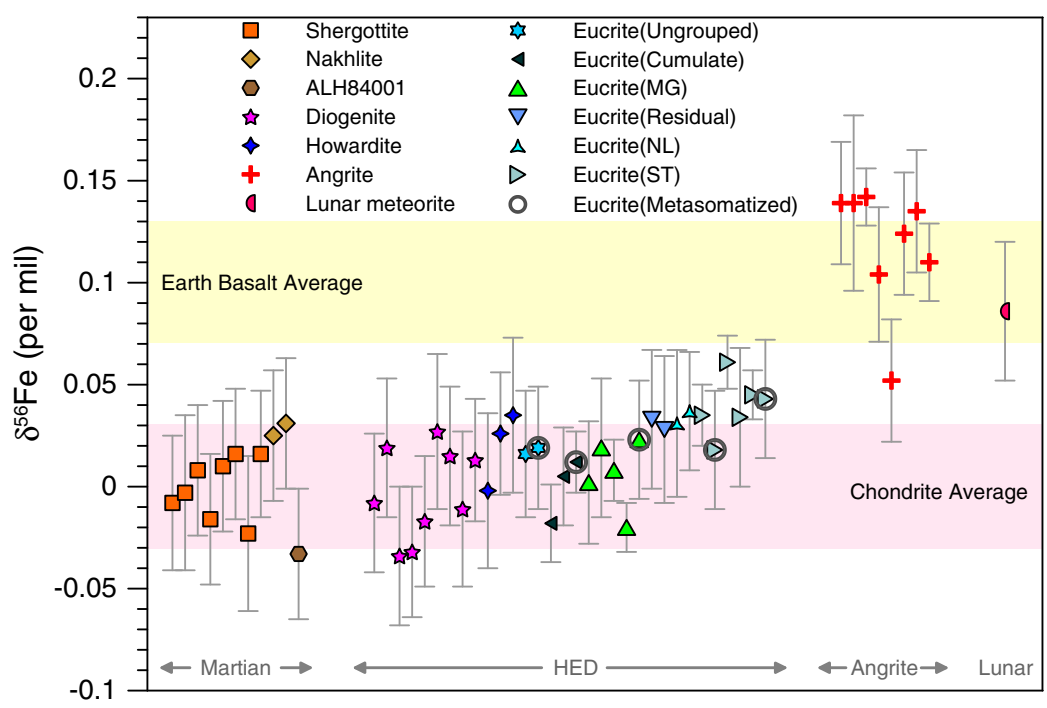

Fig. 3. Iron isotope compositions $\left(\delta^{56} \mathrm{Fe}\right)$ of martian meteorites, HEDs, and angrites. Colored shadow areas schematically show "Earth Basalt Average" and "Chondrite Average", established by a compilation of literature data (Dauphas and Rouxel, 2006; Dauphas et al., 2009a). Eucrite $(\mathrm{MG})=$ Eucrite Main Group, Eucrite (NL) = Eucrite Nuevo Laredo Trend, Eucrite (ST) = Eucrite Stannern Trend. Each point represents one meteorite. For replicate measurements in Table 1, only the average is plotted. (For interpretation of the references to color in this figure legend, the reader is referred to the web version of this article.)

$\delta^{x} \mathrm{Fe}=\left[\frac{\left({ }^{x} \mathrm{Fe} /{ }^{54} \mathrm{Fe}\right)_{\text {sample }}}{\left({ }^{x} \mathrm{Fe} /{ }^{54} \mathrm{Fe}\right)_{\text {IRMM- } 014}}-1\right] \times 1000$

where $x=56$ or 57 . In a three-isotope plot $\left(\delta^{56} \mathrm{Fe}\right.$ vs. $\delta^{57} \mathrm{Fe}$ ), all samples define a straight line of slope 1.48 (Fig. 2), consistent with mass-dependent isotope fractionation. Note that during measurements, IRMM-524a was used as bracketing standard because it has identical isotope composition to IRMM-014 and it is more readily available. The analytical uncertainties reported here for each sample are 95\% confidence intervals (see Dauphas et al. (2009b) for a detailed account on how error bars are calculated). Weighted averages and uncertainties are calculated for replicate analyses of meteorites using the following two equations:

$\bar{x}=\frac{\sum_{i=1}^{n}\left(x_{i} / e_{i}^{2}\right)}{\sum_{i=1}^{n}\left(1 / e_{i}^{2}\right)}$

$\sigma^{2}=\frac{1}{\sum_{i=1}^{n}\left(1 / e_{i}^{2}\right)}$

where $e_{i}$ is the standard deviation for each independent analysis. The uncertainties of previously published $\delta^{56} \mathrm{Fe}$ data cited in this paper are typically 2 standard deviation (2SD) or 2 standard error (2SE). Caution should be taken when comparing these weighted average uncertainties to the $2 \mathrm{SD}$ or $2 \mathrm{SE}$ in previous studies because in most instances, accuracy of the measurements has not been tested below $\pm 0.03 \%$.

\section{RESULTS}

Our iron isotope data are reported in Table 1, and illustrated in Figs. 2 and 3. The iron isotope compositions are compared to those of chondrites and terrestrial basalts, for which 10 carbonaceous chondrites define an average $\delta^{56} \mathrm{Fe}$ value of $-0.010 \%$ and Earth basalts define an average value of $\sim+0.1 \%$ relative to IRMM-014 (Craddock and Dauphas, 2011).

\subsection{Earth and Moon}

The terrestrial geostandards measured here (BIR-1, BCR-2, BHVO-2, AGV-1, AGV-2, GSP-1 and AC-E) give iron isotope compositions that are consistent with previous reports (see Craddock and Dauphas (2011) for a data compilation). The composition of BCR-2 measured here is slightly lighter than that reported by Craddock and Dauphas (2011). Three modern island arc basalts (IABs) treated with different techniques (routine, long and UTEVA chemistries) also agree with the data in a previous study (Dauphas et al., 2009a).

The iron isotope composition of the lunar highland meteorite MAC88105 is $\delta^{56} \mathrm{Fe}=+0.09 \pm 0.03 \%$, which is consistent with previously reported Apollo lunar highland rocks (Poitrasson et al., 2004; Weyer et al., 2005). Other lunar lithologies have variable iron isotope compositions that encompass this value, from $\delta^{56} \mathrm{Fe}=+0.03 \%$ for low-Ti mare basalts (Weyer et al., 2005) to $+0.27 \%$ for high-Ti mare basalts (Moynier et al., 2006). The formation of these highly fractionated iron isotope compositions in lunar samples has been discussed in several recent papers (Poitrasson et al., 2004; Weyer et al., 2005, 2007; Poitrasson, 2007; Liu et al., 2010), and will not be further addressed here.

\subsection{Martian meteorites}

The eight shergottites studied have uniform $\delta^{56} \mathrm{Fe}$ equal to $0.00 \pm 0.01 \%$. These new and more precise data are in 
agreement with previous reports (average $+0.01 \%$; Poitrasson et al., 2004; Weyer et al., 2005; Anand et al., 2006), and confirm that shergottites have an iron isotope composition identical to chondrites. The $\delta^{56} \mathrm{Fe}$ of two nakhlites $(+0.03 \pm 0.02 \%)$ and ALH84001 $(-0.03 \pm 0.03 \%)$ are also very similar to shergottites (within analytical precision). A close look at the data hints at the presence of possibly different iron isotope compositions among different groups of martian meteorites. However, these differences are at the limits of current analytical precision.

\subsection{HED meteorites}

Our new data confirm the findings of previous reports that most eucrites (cumulate eucrites, Main Group, Nuevo Laredo Trend and residual eucrites) have a chondritic iron isotope composition (average $\delta^{56} \mathrm{Fe}=0.00 \pm 0.01 \%$ ). However, it is significant that Stannern Trend (ST) eucrites (average $\delta^{56} \mathrm{Fe}=+0.05 \pm 0.01 \%$ ) are isotopically heavier than other eucrites. Our results for six individual ST eucrites are more precise than the four previous measurements, which were limited to two different ST eucrites samples: Stannern $\left(\delta^{56} \mathrm{Fe}=0.00 \pm 0.05 \%\right.$, Schoenberg and von Blanckenburg, 2006; $+0.01 \pm 0.02 \%$, Weyer et al., 2005; $-0.06 \pm 0.06 \%$, Zhu et al., 2001) and Bouvante $\left(\delta^{56} \mathrm{Fe}=+0.03 \pm 0.04 \%\right.$, Weyer et al., 2005; $+0.03 \pm$ 0.05 , Poitrasson et al., 2004). To confirm the heavier iron isotope composition of ST eucrites measured, we independently processes and analyzed the same splits at UofC using three independent chemistry methods (Table 2). All these replicated measurements of ST eucrites show consistent results (Fig. 1 and Table 1). ST eucrites are enriched in heavy iron isotopes compared to other classes of eucrites. Ungrouped eucrites, Pasamonte $(+0.02 \pm 0.03 \%)$ and NWA1240 $(+0.02 \pm 0.03 \%)$, have iron isotope compositions identical to other eucrites. Three howardites (average $\left.\delta^{56} \mathrm{Fe}=+0.02 \pm 0.02 \%\right)$ and nine diogenites $(0.00 \pm$ $0.01 \%$ ) are isotopically identical to all classes of eucrites, excluding ST. Hence, iron isotopes, unlike oxygen isotopes, cannot distinguish ungrouped eucrites from other HEDs.

\subsection{Angrites}

The eight samples of angrites (average $\delta^{56} \mathrm{Fe}=$ $+0.12 \pm 0.01 \%$ ) are isotopically heavier than chondrites, but similar to terrestrial basalts. To ensure that these data were correct, we again processed samples independently at WUSTL and UofC using three chemistry methods. All replicate measurements of the same samples processed by different methods gave the same iron isotope compositions within analytical uncertainty (Table 1 and Fig. 1) and confirm that angrites have heavy fractionated iron isotope compositions.

\section{DISCUSSION}

In the following section, we discuss the implications of the enrichment in the heavy isotopes of Fe observed in angrites and Stannern Trend (ST) eucrites in terms of the redox state of the parent bodies and magmatic differentiation.

\subsection{Redox-controlled iron isotope fractionation on the angrite parent-body?}

Several processes can be considered in order to explain the fractionated iron isotope composition of basaltic angrites compared to other basaltic meteorites, including fractionation during low temperature terrestrial alteration, metal-silicate segregation, mineral fractionation, volatilization during impact, and partial silicate mantle melting. All angrites studied here are finds from the Sahara desert, Antarctica or Argentina. Leaching of isotopically light iron during terrestrial weathering can raise the $\delta^{56} \mathrm{Fe}$ value of the residual rock up to $+0.1 \%$ (Saunier et al., 2010). This process, however, is an unlikely candidate to explain the enrichment of heavy isotopes in angrites because all the angrites have a very narrow range of $\delta^{56} \mathrm{Fe}(+0.12$ $\pm 0.01 \%$ oo. These samples were found across a wide range of environments (from icy to hot desert) and have been subject to different exposure histories, which would not fractionate iron isotopes during alteration to an almost identical extent. In addition, for all other achondrite groups measured in this study, there is no difference in iron isotope composition between meteorite falls and finds indicating that terrestrial exposure has not affected isotope behavior for the samples in this study.

Equilibrium iron isotope fractionation between metal core and mantle is also not a viable explanation for the fractionated iron isotope compositions of angrites. High temperature $\left(2000^{\circ} \mathrm{C}\right)$ and high pressure $(7.7 \mathrm{GPa})$ equilibrium melting experiments of chondritic materials show no iron isotope fractionation between metal alloy and silicate melt (Poitrasson et al., 2009). Low-temperature, low-pressure metal silicate segregation would enriched the metal in heavy isotopes and the silicates in lighter isotopes (as seen in pallasites by Poitrasson et al. (2005)). Campbell and Humayun (2005) proposed that IVB iron meteorites, enriched in heavy iron isotopes (Williams et al., 2006), could be the core of the angrite parent-body. If this is the case, angrite is expected to be enriched in light iron isotopes, which is the inverse of what is observed in angrites. Only at extremely high pressures $(>100 \mathrm{GPa})$ encountered at the core-mantle boundary of the Earth, metallic core could be enriched in light iron isotopes while silicate mantle could be enriched heavy iron isotopes according to theoretical calculations (Polyakov, 2009). Although the parent-body of angrites is still unidentified; there is little doubt that these meteorites come from a small-scale asteroid (Burbine et al., 2006; Rivkin et al., 2007; Trilling et al., 2007).

Preferential evaporation of lighter $\mathrm{Fe}$ during impact events (and enrichment in the heavier in the residue) is a mechanism that has been proposed to explain the difference between terrestrial and lunar basalts (Poitrasson et al., 2004). All angrites (except Angra dos Reis and NWA2999) are unshocked and do not show particular impact features (Scott and Bottke, 2011). In addition, angrites are depleted in moderately volatile elements, but they are not notably more depleted in moderately volatile elements (e.g., $\mathrm{Zn}$, Cd) than eucrites (Weisberg et al., 2006). If volatilization due to impacts was the origin of the fractionation observed in angrites, similar effects should be found in eucrites, which 

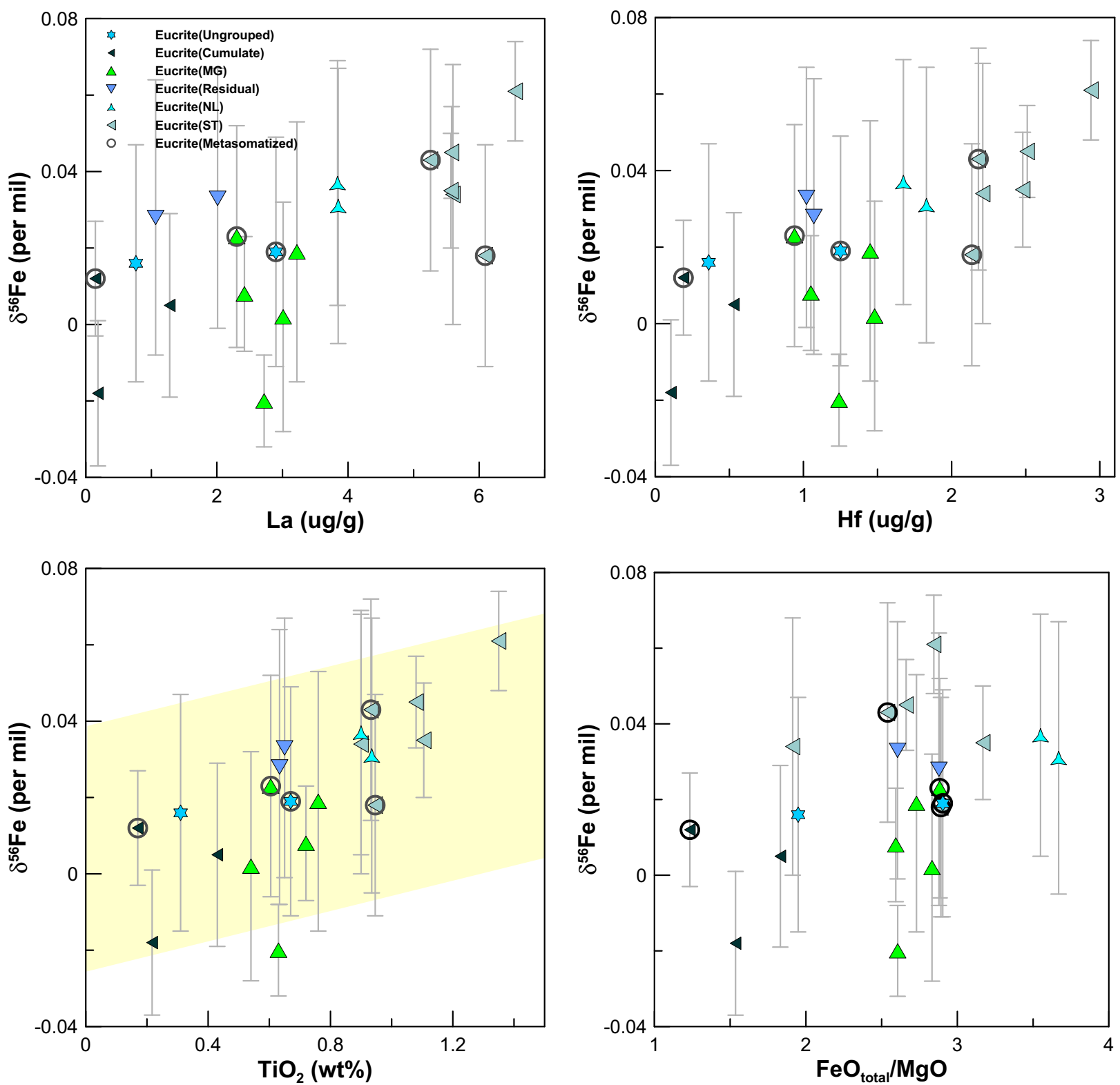

Fig. 4. Iron isotope compositions $\left(\delta^{56} \mathrm{Fe}\right)$ vs. $\mathrm{La}, \mathrm{Hf}, \mathrm{TiO}_{2}$ and $\mathrm{FeO}_{\text {total }} / \mathrm{MgO}$. The yellow shaded area represents ilmenite-controlled iron isotope fractionation (see text for details). Eucrite $(\mathrm{MG})=$ Eucrite Main Group, Eucrite $(\mathrm{NL})=$ Eucrite Nuevo Laredo Trend, Eucrite $(\mathrm{ST})=$ Eucrite Stannern Trend. Each point represents one meteorite. For replicate measurements in Table 1, only the average is plotted. Data sources: The concentration data of Pasamonte, Moore County, Cachari, Jonzac, Serra de Magé, and Camel Donga come from Barrat et al. (2000); Nuevo Laredo, Juvinas, Stannern, NWA4523, and Bouvante from Barrat et al. (2007b); NWA049 and NWA1240 from Barrat et al. (2003); Sahara 02501 from Barrat et al. (2007a); NWA2061 from Barrat et al. (2011); Yamato75011 from Fukuoka and Ikeda (1983); Agoult and Dag945 from Yamaguchi et al. (2009); EET87548 comes from Warren et al. (2009); Pomozdino from Kitts and Lodders (1998). (For interpretation of the references to color in this figure legend, the reader is referred to the web version of this article.)

is not the case. Therefore the enrichment in the heavier iron isotopes of angrites is unlikely caused by volatilization during impact processing, although this cannot be definitely ruled out.

Redox-controlled iron isotope fractionation through partial mantle melting provides one possible explanation for the fractionated iron isotope compositions of angrites. Redox proxies indicate that angrites probably formed in a relatively oxidized environment (IW +1 to +2 ; Jurewicz et al., 1991, 1993; McKay et al., 1994), even though the exact redox conditions are difficult to quantify. Dauphas et al. (2009a) devised a quantitative model of iron isotope fractionation between source and melt as a function of $\mathrm{Fe}(\mathrm{III}) / \mathrm{Fe}(\mathrm{II})$ ratio. According to this model, given the low $\mathrm{Fe}(\mathrm{III}) / \mathrm{Fe}(\mathrm{II})$ ratio of angrites, little $\mathrm{Fe}$ isotope fractionation would be expected during partial melting. How- 
ever, the exact redox condition of the mantle of angrite's parent body remains poorly known and it is also uncertain whether redox conditions can affect iron equilibrium isotope fractionation factors by modifying the structure of the melt even in systems with little Fe(III). Further work remains to be done to understand iron isotopic fractionation in angrites.

\subsection{Iron isotope fractionation on the HED parent-body}

Petrological arguments indicate that HED meteorites were formed in a low oxygen fugacity environment, close to the IW buffer, with Fe(III) absent (Stolper, 1977; Hewins and Ulmer, 1984). The chondritic $\delta^{56} \mathrm{Fe}$ values of diogenites, howardites, cumulate eucrites, MG-NL eucrites, and residual eucrites are consistent with a chondritic iron isotope composition of the HED parent-body mantle that has not been modified by core segregation or partial melting, as is also seen on Mars (Poitrasson et al., 2004).

The $\delta^{56} \mathrm{Fe}$ values of ST eucrites are higher than those of other eucrites. ST eucrites do not show any particular depletion in volatile elements or impact features when compared to other eucrites (Barrat et al., 2007b). Therefore, volatilization of light isotopes of Fe due to impact is a very unlikely origin for iron isotope difference between ST eucrites and other eucrites. Iron isotope fractionation is also observed in lunar basalts where redox state is extremely low (IW-2 to -1 ; Liu et al., 2010). It has been proposed that ilmenite fractionation controls the $0.1 \%$ difference in $\delta^{56} \mathrm{Fe}$ between low-Ti and high-Ti lunar mare basalts (Craddock et al., 2010). Ilmenite is enriched in the heavy isotopes of iron between $0.16 \%$ and $0.42 \%$ in $\delta^{56} \mathrm{Fe}$ (Craddock et al., 2010). ST eucrites are richer in Ti than the other eucrites and petrographic studies have also shown there is relatively more ilmenite in ST eucrites than in other eucrites (0.93-1.20 vol \% vs. trace-1.00 vol \%, respectively; Delaney et al., 1984; Warren et al., 1990). By mixing ilmenite with $\delta^{56} \mathrm{Fe}=+0.42 \%$ (the maximum fractionation) to main-group or cumulate eucrites $\left(\delta^{56} \mathrm{Fe} \approx 0\right)$, we obtained a $\delta^{56} \mathrm{Fe}=$ $0.02 \%$ (see Appendix for the mixing calculation; the calculation results are plotted as the shaded area in Fig. 4). This could be enough to explain the observed ST eucrites values within analytical errors $( \pm 0.03 \%$ ). If the ilmenites have a lower $\delta^{56} \mathrm{Fe}$, only a fraction of the isotopic effect observed in ST eucrites can be explained by ilmenites and other source of isotope fractionation cannot be excluded. It is possible that other Fe-bearing minerals also controlled the iron isotope composition of eucrites, and further work is needed to characterize $\mathrm{Fe}$ isotope fractionation between minerals in eucrite meteorites.

The origin of ST eucrites is debated (see review by McSween et al. (2010)). ST eucrites are petrographically and geochemically similar to MG eucrites, but are enriched in incompatible elements (Fig. 4). Their origin was first explained as smaller degrees of partial melting of the same source as other eucrites (Consolmagno and Drake, 1977; Stolper, 1977; Hsu and Crozaz, 1996). However, this model is not consistent with the growing database for trace lithophile and siderophile elements (Mittlefehldt and Lindstrom, 2003; Barrat et al., 2007b). It has been proposed recently that ST eucrites are derived from the same parent magma to $\mathrm{MG}$ eucrites and have been modified by the assimilation of the asteroidal crust (Barrat et al., 2007b). This model is supported by our iron isotope data. It is observed in this study that $\delta^{56} \mathrm{Fe}$ is correlated with Ti and other incompatible elements (Fig. 4). Non-modal re-melting of previously formed asteroidal crust would preferentially consume ilmenite (Barrat et al., 2007b). Partial melts formed from the eucritic crust will be rich not only in Ti, but also in the heavy isotopes of $\mathrm{Fe}$ because ilmenite in the case of the Moon has been demonstrated to be consistently heavy by up to $0.42 \%$ in $\delta^{56} \mathrm{Fe}$ (Craddock et al., 2010). Contamination of ordinary basaltic eucrites by such components could explain the origin of ST eucrites with fractionated iron isotope compositions.

\subsection{Iron isotopes and fluid-rock interactions on asteroidal bodies}

A few eucrites display pre-terrestrial secondary minerals, which may have recorded interactions with fluids. Serra de Magé contains quartz veinlets, which have been ascribed by Treiman et al. (2004) to the circulation of water. A few other eucrites display Fe-enrichment along the cracks that crosscut the pyroxenes, and sometimes deposits of Fe-rich olivine and anorthitic plagioclase inside the fractures (Barrat et al., 2011). The origin of these phases is a matter of debate. Barrat et al. (2011) have proposed that this Feenrichment is the result of a metasomatic event. The nature of the metasomatic agent is not clear, but could have been an aqueous fluid. Subsequently, Roszjar et al. (2011) proposed that Fe-olivine and anorthite were formed by incongruent in situ melting of pyroxene at a temperature slightly above the temperature of formation of the primary pyroxenes, at about $1150^{\circ} \mathrm{C}$.

Fluid-rock interaction and exsolution of aqueous fluids could preferentially remove light iron isotopes from bulk rock and is an important mechanism for iron chemical and isotope fractionation in both low- and high-temperature processes on Earth (Rouxel et al., 2003; Poitrasson and Freydier, 2005; Chapman et al., 2009). Thus, the fingerprint of aqueous fluid interaction in eucritic rocks could be detected using $\mathrm{Fe}$ isotopes. Among our samples, five eucrites have been suspected to interact with aqueous fluids: Serra de Magé, Pasamonte, NWA049, NWA2061 and Y-75011 (circled points in Figs. 3 and 4). None of these samples show iron isotope compositions fractionated relative to other similarly grouped eucrites. These results do not rule out definitively the involvement of fluids during eucrite evolution, but imply that fluid interactions on eucritic parent-bodies had negligible impact on iron isotope compositions. These results exclude the possibility that secondary processes on HED parent-body are the reason for the fractionated iron isotope compositions of ST eucrites.

\section{CONCLUSION}

We have found that $\mathrm{Fe}$ is enriched in the heavier isotopes in angrites (by $+0.12 \pm 0.01 \%$; similar to terrestrial 
Table A1

Parameters of the mass-balance used in Eq. (A1) to calculate the ilmenite-controlled iron isotope fractionation.

\begin{tabular}{|c|c|c|c|}
\hline Parameter & Meaning & Value & Reference \\
\hline$A_{\text {cumulate_MG }}$ & $\begin{array}{l}\text { Ilmenite abundance in eucrite (cumulate) } \\
\text { and eucrite (MG) (vol \%) }\end{array}$ & Trace-1.00 & Delaney et al. (1984) \\
\hline$A_{\mathrm{ST}}$ & $\begin{array}{l}\text { Ilmenite abundance in eucrite (ST) } \\
(\text { vol \%) }\end{array}$ & $0.93-1.20$ & Delaney et al. (1984) and Warren et al. (1990) \\
\hline$\rho_{\text {ilmenite }}$ & Ilmenite density $\left(\mathrm{g} / \mathrm{cm}^{3}\right)$ & 4.79 & Lodders and Fegley (1998) \\
\hline$\rho_{\text {eucrite }}$ & Eucrite average density $\left(\mathrm{g} / \mathrm{cm}^{3}\right)$ & 3.20 & Kitts and Lodders (1998) \\
\hline$C_{\text {ilmenite }}$ & Iron concentration in ilmenite $(\%)$ & 36.81 & Ilmenite $\left(\mathrm{FeTiO}_{3}\right)$ stoichiometric value \\
\hline$C_{\mathrm{ST}}$ & $\begin{array}{l}\text { Iron concentration in } \\
\text { eucrite }(\mathrm{ST})(\%)\end{array}$ & $13.72-15.27$ & Kitts and Lodders (1998) \\
\hline$\delta^{56} \mathrm{Fe}_{\text {ilmenite }}$ & $\begin{array}{l}\text { Iron isotope composition of } \\
\text { separated ilmenite }\end{array}$ & $0.16-0.42$ & Craddock et al. (2010) \\
\hline$\delta^{56} \mathrm{Fe}_{\text {cumulate_MG }}$ & $\begin{array}{l}\text { Average iron isotope composition } \\
\text { of eucrite (cumulate) and } \\
\text { eucrite (MG) }\end{array}$ & $\sim 0.00$ & $\begin{array}{l}\text { This study and previous references } \\
\text { (Poitrasson et al., 2004; Weyer et al., 2005; } \\
\text { Schoenberg and von Blanckenburg, 2006) }\end{array}$ \\
\hline
\end{tabular}

Notes: Eucrite $(\mathrm{MG})=$ Eucrite Main Group, Eucrite $(\mathrm{ST})=$ Eucrite Stannern Trend.

basalts) and in Stannern Trend eucrites (by $+0.05 \pm$ $0.01 \%$ ). Martian and HEDs (except Stannern Trend) meteorites have iron isotope compositions identical to chondrites. Isotope fractionations during magmatic processes (partial melting, mineral fractionation and fluid exsolution) under different redox conditions are the best explanations to explain iron isotope variations in planetary crusts. However, further work remains to be done to document experimentally equilibrium iron isotope fractionation between melts and minerals to understand iron isotope variations in magmatic rocks. Our results show that not only terrestrial and lunar basalts are isotopically fractionated in iron but also crustal rocks from at least two asteroids with different sizes and volatilization histories.

\section{ACKNOWLEDGMENTS}

We acknowledge the support of NASA LASER (NNX09AM64G) and EXO (NNX12AD88G) to F.M.; NASA Cosmochemistry (NNX09AG59G), NSF Petrology and Geochemistry (EAR-0820807) and a Packard Fellowship to N.D. We also are indebted to Joseph Boesenberg and Denton Ebel (American Museum of National History, New York), Timothy McCoy (US National Museum of Natural History, Smithsonian Institution, Washington, DC), Caroline Smith (The Natural History Museum, London), Alex Bevan (Western Australian Museum, Perth), Franz Brandstatter (Naturhistorisches Museum, Vienna), James Holstein, Clarita Nunez and Philip Heck (The Field Museum, Chicago), Meenakshi Wadhwa (Arizona State University, Tempe), Cecilia Satterwhite (NASA Johnson Space Center, Houston) and the Comité de Gestion (Museum Nationale d'Histoire Naturelle, Paris) and Guy Consolmagno (Vatican) for their generous donations of meteorite samples for this work and their confidence in our analytical and scientific capabilities. Julien Foriel and Randy Paniello are acknowledged for the help in the lab at WashU. KW thanks Francis Albarède, Robert Criss, Bruce Fegley, Bradley Jolliff, Randy Korotev, and Frank Podosek for discussions. Franck Poitrasson, Stefan Weyer and an anonymous reviewer are thanked for their reviews and excellent comments. The authors also thank Sara Russell for handling and editing the manuscript.

\section{APPENDIX A}

Ilmenite is enriched in the heavy isotopes of iron between $0.16 \%$ and $0.42 \%$ in $\delta^{56} \mathrm{Fe}$ (Craddock et al., 2010). By using the $\delta^{56} \mathrm{Fe}$ value of separated ilmenite and the modal abundance of ilmenite in ST eucrites, we can construct a simplified mixing model of ilmenite-controlled iron isotope fractionation (Eq. (A1)).

$\delta^{56} \mathrm{Fe}_{\mathrm{ST}}=\delta^{56} \mathrm{Fe}_{\text {cumulate_MG }}+\Delta_{\text {ilmenite }} \cdot \delta^{56} \mathrm{Fe}_{\text {ilmenite }}$

$\delta^{56} \mathrm{Fe}_{\mathrm{ST}}$ is the iron isotope composition of ST eucrites; $\delta^{56} \mathrm{Fe}_{\text {cumulate } \mathrm{MG}}$ is the iron isotope composition of cumulate or MG eucrites; $\delta^{56} \mathrm{Fe}_{\mathrm{ST}}$ is the iron isotope composition of mineral ilmenite. $\Delta_{\text {ilmenite }}$ is the enrichment of ilmenite in ST eucrites compared to cumulate or MG eucrites; $\Delta_{\text {ilmenite }}$ is a function of the abundance of ilmenite in ST and in cumulate or MG eucrites. Considering the conversion of volume percentage reported in references to weight percentage, and iron concentration in mineral ilmenite and in bulk eucrites, we could write:

$\Delta_{\text {ilmenite }}=\left(A_{\mathrm{ST}}-A_{\text {cumulate_MG }}\right) \cdot \frac{\rho_{\text {ilmenite }}}{\rho_{\text {eucrite }}} \cdot \frac{C_{\text {ilmenite }}}{C_{\mathrm{ST}}}$

The meanings and values of the parameters used in Eq. (A2) are listed in Table A1.

Hence, Eq. (A1) can be developed into Eq. (A3):

$$
\begin{aligned}
\delta^{56} \mathrm{Fe}_{\mathrm{ST}}= & \delta^{56} \mathrm{Fe}_{\text {cumulate_MG }}+\left(A_{\mathrm{ST}}-A_{\text {cumulate_MG }}\right) \\
& \cdot \frac{\rho_{\text {ilmenite }}}{\rho_{\text {eucrite }}} \cdot \frac{C_{\text {ilmenite }}}{C_{\mathrm{ST}}} \cdot \delta^{56} \mathrm{Fe}_{\text {ilmenite }}
\end{aligned}
$$

By using the maximum fractionation value $\left(\delta^{56} \mathrm{Fe}=\right.$ $0.42 \%$ ) measured in ilmenite (Craddock et al., 2010), we obtained a $\delta^{56} \mathrm{Fe}=0.02$ for ST eucrites, which could largely explain the difference between ST eucrites and cumulate or MG eucrites within analytical errors $( \pm 0.03 \%)$. However, if we use the minimum fractionation value $\left(\delta^{56} \mathrm{Fe}=0.16 \%\right.$ o $)$ measured in ilmenite (Craddock et al., 2010), we obtained a $\delta^{56} \mathrm{Fe}=0.01$ for $\mathrm{ST}$ eucrites. In this case, other minerals 
might also contribute to the enrichment of iron isotope in ST eucrites. Due to the lack of iron isotope data of relevant minerals, there is no further constraint on this problem.

\section{REFERENCES}

Allègre C. J., Poirier J.-P., Humler E. and Hofmann A. W. (1995) The chemical composition of the Earth. Earth Planet. Sci. Lett. 134, 515-526.

Anand M., Russell S. S., Blackhurst R. L. and Grady M. M. (2006) Searching for signatures of life on Mars: an Fe-isotope perspective. Philos. Trans. R. Soc. Lond. B Biol. Sci. 361, 1715-1720.

Baker J., Bizzarro M., Wittig N., Connelly J. and Haack H. (2005) Early planetesimal melting from an age of $4.5662 \mathrm{Gyr}$ for differentiated meteorites. Nature 436, 1127-1131.

Barrat J. A., Blichert-Toft J., Gillet P. and Keller F. (2000) The differentiation of eucrites: the role of in situ crystallization. Meteorit. Planet. Sci. 35, 1087-1100.

Barrat J. A., Jambon A., Bohn M., Blichert-Toft J., Sautter V., Göpel C., Gillet P., Boudouma O. and Keller F. (2003) Petrology and geochemistry of the unbrecciated achondrite Northwest Africa 1240 (NWA 1240): an HED parent body impact melt. Geochim. Cosmochim. Acta 67, 3959-3970.

Barrat J. A., Yamaguchi A., Greenwood R. C., Bohn M., Cotten J., Benoit M. and Franchi I. A. (2007a) Could Stannern-trend eucrites be crustal-contaminated melts? Lunar Planet. Sci. Conf. 38, \#1558 (abstr.).

Barrat J. A., Yamaguchi A., Greenwood R. C., Bohn M., Cotten J., Benoit M. and Franchi I. A. (2007b) The Stannern trend eucrites: contamination of main group eucritic magmas by crustal partial melts. Geochim. Cosmochim. Acta 71, 4108-4124.

Barrat J. A., Yamaguchi A., Greenwood R. C., Benoit M., Cotten J., Bohn A. and Franchi I. A. (2008) Geochemistry of diogenites: still more diversity in their parental melts. Meteorit. Planet. Sci. 43, 1759-1775.

Barrat J. A., Yamaguchi A., Bunch T. E., Bohn M., Bollinger C. and Ceuleneer G. (2011) Possible fluid-rock interactions on differentiated asteroids recorded in eucritic meteorites. Geochim. Cosmochim. Acta 75, 3839-3852.

Belshaw N. S., Zhu X. K., Guo Y. and O'Nions R. K. (2000) High precision measurement of iron isotopes by plasma source mass spectrometry. Int. J. Mass Spectrom. 197, 191-195.

Bouvier A., Brennecka G. A., Sanborn M. E. and Wadhwa M. (2011) The formation of the angritic crust. Goldschmidt Conference (abstr.).

Burbine T. H., McCoy T. J., Hinrichs J. L. and Lucey P. G. (2006) Spectral properties of angrites. Meteorit. Planet. Sci. 41, 11391145.

Campbell A. J. and Humayun M. (2005) Compositions of group IVB iron meteorites and their parent melt. Geochim. Cosmochim. Acta 69, 4733-4744.

Chapman J. B., Weiss D. J., Shan Y. and Lemburger M. (2009) Iron isotope fractionation during leaching of granite and basalt by hydrochloric and oxalic acids. Geochim. Cosmochim. Acta 73, 1312-1324.

Consolmagno G. J. and Drake M. J. (1977) Composition and evolution of the eucrite parent body: evidence from rare earth elements. Geochim. Cosmochim. Acta 41, 1271-1282.

Craddock P. R. and Dauphas N. (2011) Iron isotopic compositions of geological reference materials and chondrites. Geostand. Geoanal. Res. 35, 101-123.

Craddock P. R., Dauphas N. and Clayton R. N. (2010) Mineralogical control on iron isotopic fractionation during lunar differentiation and magmatism. Lunar Planet. Sci. Conf. 41, \#1230 (abstr.).
Dauphas N. and Rouxel O. (2006) Mass spectrometry and natural variations of iron isotopes. Mass Spectrom. Rev. 25, 515-550.

Dauphas N. and Chaussidon M. (2011) A perspective from extinct radionuclides on a young stellar object: the Sun and its accretion disk. Annu. Rev. Earth Planet. Sci. 39, 351-386.

Dauphas N., Janney P. E., Mendybaev R. A., Wadhwa M., Richter F. M., Davis A. M., van Zuilen M., Hines R. and Foley C. N. (2004) Chromatographic separation and multicollectionICPMS analysis of iron. Investigating mass-dependent and independent isotope effects. Anal. Chem. 76, 5855-5863.

Dauphas N., Craddock P. R., Asimow P. D., Bennett V. C., Nutman A. P. and Ohnenstetter D. (2009a) Iron isotopes may reveal the redox conditions of mantle melting from Archean to Present. Earth Planet. Sci. Lett. 288, 255-267.

Dauphas N., Pourmand A. and Teng F. Z. (2009b) Routine isotopic analysis of iron by HR-MC-ICPMS: how precise and how accurate? Chem. Geol. 267, 175-184.

Day J. M. D., Taylor L. A., Floss C. and McSween H. Y. (2006) Petrology and chemistry of MIL 03346 and its significance in understanding the petrogenesis of nakhlites on Mars. Meteorit. Planet. Sci. 41, 581-606.

Delaney J. S., Prinz M. and Takeda H. (1984) The polymict eucrites. J. Geophys. Res. 89, C251-C288.

Drake M. J. (2001) The eucrite/Vesta story. Meteorit. Planet. Sci. 36, 501-513.

Duke M. B. and Silver L. T. (1967) Petrology of eucrites, howardites and mesosiderites. Geochim. Cosmochim. Acta 31, $1637-1665$.

Fukuoka T. and Ikeda K. (1993) Chemical compositions of 5 Yamato encrites. Papers 8th NIPR Symposium on Antarctic Meteorites, pp. 49-50.

Gellissen M., Palme H., Korotev R. L. and Irving A. J. (2007) NWA2999, a unique angrite with a large chondritic component. Lunar Planet. Sci. 38, \#1612 (abstr.).

Gillet P., Barrat J. A., Beck P., Marty B., Greenwood R. C., Franchi I. A., Bohn M. and Cotten J. (2005) Petrology, geochemistry, and cosmic-ray exposure age of lherzolitic shergottite Northwest Africa 1950. Meteorit. Planet. Sci. 40, 1175-1184.

Heimann A., Beard B. L. and Johnson C. M. (2008) The role of volatile exsolution and sub-solidus fluid/rock interactions in producing high $56 \mathrm{Fe} / 54 \mathrm{Fe}$ ratios in siliceous igneous rocks. Geochim. Cosmochim. Acta 72, 4379-4396.

Hewins R. H. and Ulmer G. C. (1984) Intrinsic oxygen fugacities of diogenites and mesosiderite clasts. Geochim. Cosmochim. Acta 48, 1555-1560.

Hin R. C., Schimidt M. W., Wiederhold J. G. and Bourdon B. (2010) Constraints on $\mathrm{Fe}$ isotope fractionation between liquid metal and liquid silicate from experiments at $1 \mathrm{GPa}$ and 1250 $1300{ }^{\circ} \mathrm{C}$. American Geophysical Union, Fall Meeting, \#V44B-03 (abstr.).

Horwitz E. P., Dietz M. L., Chiarizia R., Diamond H., Essling A. M. and Graczyk D. (1992) Separation and preconcentration of uranium from acidic media by extraction chromatography. Anal. Chim. Acta 266, 25-37.

Hsu W. B. and Crozaz G. (1996) Mineral chemistry and the petrogenesis of eucrites. 1. Noncumulate eucrites. Geochim. Cosmochim. Acta 60, 4571-4591.

Humayun M. and Clayton R. N. (1995) Potassium isotope cosmochemistry - genetic-implications of volatile element depletion. Geochim. Cosmochim. Acta 59, 2131-2148.

Irving A. J. and Kuehner S. M. (2007) Plutonic angrite NWA 4801 and a model for the angrite parent body consistent with petrological and chronological constraints. Workshop on the chronology of meteorites and the early Solar System, \#4050 (abstr.). 
Jambon A., Barrat J. A., Boudouma O., Fonteilles M., Badia D., Göpel C. and Bohn M. (2005) Mineralogy and petrology of the angrite Northwest Africa 1296. Meteorit. Planet. Sci. 40, 361375.

Jambon A., Boudouma O., Fonteilles M., Guillou C. L., Badia D. and Barrat J. A. (2008) Petrology and mineralogy of the angrite Northwest Africa 1670. Meteorit. Planet. Sci. 43, 1783-1795.

Jarosewich E. (1990) Chemical-analyses of meteorites - a compilation of stony and iron meteorite analyses. Meteoritics 25, 323337.

Jolliff B. L., Korotev R. L. and Haskin L. A. (1991) A ferroan region of the lunar highlands as recorded in meteorites MAC88104 and MAC88105. Geochim. Cosmochim. Acta 55, 3051-3071.

Jurewicz A. J. G., Mittlefehldt D. W. and Jones J. H. (1991) Partial melting of the Allende (CV3) meteorite - implications for origins of basaltic meteorites. Science 252, 695-698.

Jurewicz A. J. G., Mittlefehldt D. W. and Jones J. H. (1993) Experimental partial melting of the allende (CV) and murchison (CM) chondrites and the origin of asteroidal basalts. Geochim. Cosmochim. Acta 57, 2123-2139.

Kitts K. and Lodders K. (1998) Survey and evaluation of eucrite bulk compositions. Meteorit. Planet. Sci. 33, A197-A213.

Koeberl C., Kurat G. and Brandstatter F. (1991) MAC88105 - a regolith breccia from the lunar highlands: mineralogical, petrological, and geochemical studies. Geochim. Cosmochim. Acta 55, 3073-3087.

Korotev R. L., Jolliff B. L., Zeigler R. A., Gillis J. J. and Haskin L. A. (2003) Feldspathic lunar meteorites and their implications for compositional remote sensing of the lunar surface and the composition of the lunar crust. Geochim. Cosmochim. Acta 67, 4895-4923.

Kuehner S. M., Irving A. J., Bunch T. E., Wittke J. H., Hupé G. M. and Hupé A. C. (2006) Coronas and symplectites in plutonic angrite NWA 2999 and implications for Mercury as the angrite parent body. Lunar Planet. Sci. Conf. 37, \#1344 (abstr.).

Lindstrom M. M., Wentworth S. J., Martinez R. R., Mittlefehldt D. W., McKay D. S., Ming-Sheng W. and Lipschutz M. E. (1991) Geochemistry and petrography of the MacAlpine Hills lunar meteorites. Geochim. Cosmochim. Acta 55, 3089-3103.

Liu Y., Spicuzza M. J., Craddock P. R., Day J. M. D., Valley J. W., Dauphas N. and Taylor L. A. (2010) Oxygen and iron isotope constraints on near-surface fractionation effects and the composition of lunar mare basalt source regions. Geochim. Cosmochim. Acta 74, 6249-6262.

Lodders K. (1998) A survey of shergottite, nakhlite and chassigny meteorites whole-rock compositions. Meteorit. Planet. Sci. 33, A183-A190.

Lodders K. and Fegley B. (1998) The Planetary Scientist's Companion. Oxford University Press, New York.

McCammon C. (2005) The paradox of mantle redox. Science 308, 807-808.

McCarthy T. S., Ahrens L. H. and Erlank A. J. (1972) Further evidence in support of the mixing model for howardite origin. Earth Planet. Sci. Lett. 15, 86-93.

McCord T. B., Adams J. B. and Johnson T. V. (1970) Asteroid Vesta: spectral reflectivity and compositional implications. Science 168, 1445-1447.

McKay G., Lindstrom D., Yang S.-R. and Wagstaff J. (1988) Petrology of unique achondrite Lewis Cliff 86010. Lunar Planet. Sci. Conf. 19, \#762 (abstr.).

McKay G., Le L., Wagstaff J. and Crozaz G. (1994) Experimental partitioning of rare-earth elements and strontium - constraints on petrogenesis and redox conditions during crystallization of antarctic angrite lewis cliff-86010. Geochim. Cosmochim. Acta 58, 2911-2919.
McSween H., Mittlefehldt D., Beck A., Mayne R. and McCoy T. (2010) HED meteorites and their relationship to the geology of Vesta and the Dawn mission. Space Sci. Rev., 1-34.

Millet M.-A., Baker J. A. and Payne C. E. (2012) Ultra-precise stable $\mathrm{Fe}$ isotope measurements by high resolution multiplecollector inductively coupled plasma mass spectrometry with a $57 \mathrm{Fe}-58 \mathrm{Fe}$ double spike. Chem. Geol. 304-305, 18-25.

Mittlefehldt D. W. and Lindstrom M. M. (2003) Geochemistry of eucrites: genesis of basaltic eucrites, and $\mathrm{Hf}$ and $\mathrm{Ta}$ as petrogenetic indicators for altered antarctic eucrites. Geochim. Cosmochim. Acta 67, 1911-1934.

Mittlefehldt D. W., McCoy T. J., Goodrich C. A. and Kracher A. (1998) Non-chondritic meteorites from asteroidal bodies. In Planetary Materials (ed. J. J. Papike). Mineralogical Society of America. Reviews in Mineralogy 36, p. 195.

Mittlefehldt D. W., Killgore M. and Lee M. T. (2002) Petrology and geochemistry of D'Orbigny, geochemistry of Sahara 99555, and the origin of angrites. Meteorit. Planet. Sci. 37, 345-369.

Moynier F., Albarède F. and Herzog G. F. (2006) Isotopic composition of zinc, copper, and iron in lunar samples. Geochim. Cosmochim. Acta 70, 6103-6117.

Neal C. R., Taylor L. A., Lui Y.-g. and Schmitt R. A. (1991) Paired lunar meteorites MAC88104 and MAC88105: a new "fan" of lunar petrology. Geochim. Cosmochim. Acta 55, 3037-3049.

Nyquist L. E., Kleine T., Shih C. Y. and Reese Y. D. (2009) The distribution of short-lived radioisotopes in the early solar system and the chronology of asteroid accretion, differentiation, and secondary mineralization. Geochim. Cosmochim. Acta 73, $5115-5136$.

Poitrasson F. (2007) Does planetary differentiation really fractionate iron isotopes? Earth Planet. Sci. Lett. 256, 484-492.

Poitrasson F. and Freydier R. (2005) Heavy iron isotope composition of granites determined by high resolution MC-ICP-MS. Chem. Geol. 222, 132-147.

Poitrasson F., Halliday A. N., Lee D. C., Levasseur S. and Teutsch N. (2004) Iron isotope differences between Earth, Moon, Mars and Vesta as possible records of contrasted accretion mechanisms. Earth Planet. Sci. Lett. 223, 253-266.

Poitrasson F., Levasseur S. and Teutsch N. (2005) Significance of iron isotope mineral fractionation in pallasites and iron meteorites for the core-mantle differentiation of terrestrial planets. Earth Planet. Sci. Lett. 234, 151-164.

Poitrasson F., Roskosz M. and Corgne A. (2009) No iron isotope fractionation between molten alloys and silicate melt to 2000 degrees $\mathrm{C}$ and $7.7 \mathrm{GPa}$ : experimental evidence and implications for planetary differentiation and accretion. Earth Planet. Sci. Lett. 278, 376-385.

Polyakov V. B. (2009) Equilibrium iron isotope fractionation at core-mantle boundary conditions. Science 323, 912-914.

Polyakov V. B. and Mineev S. D. (2000) The use of Mossbauer spectroscopy in stable isotope geochemistry. Geochim. Cosmochim. Acta 64, 849-865.

Prinz M., Weisberg M. K. and Nehru C. E. (1988) LEW 86010, a second angrite: relationship to CAl's and opaque matrix. Lunar Planet. Sci. Conf. 19, \#949 (abstr.).

Reid A. M. and Barnard B. M. (1979) Unequilibrated and equilibrated eucrites. Lunar Planet. Sci. 10, \#1019 (abstr.).

Rivkin A. S., Trilling D. E., Thomas C. A., DeMeo F., Spahr T. B. and Binzel R. P. (2007) Composition of the L5 Mars Trojans: neighbors, not siblings. Icarus 192, 434-441.

Roszjar J., Metzler K., Bischoff A., Barrat J., Geisler T., Greenwood R. C., Franchi I. A. and Klemme S. (2011) Thermal history of Northwest Africa (NWA) 5073 - a coarsegrained Stannern-trend eucrite containing $\mathrm{cm}$-sized pyroxenes and large zircon grains. Meteor. Planet. Sci. 46, 1754-1773. 
Rouxel O., Dobbek N., Ludden J. and Fouquet Y. (2003) Iron isotope fractionation during oceanic crust alteration. Chem. Geol. 202, 155-182.

Rudnick R. L. and Gao S. (2003) The composition of the continental crust. In Treatise on Geochemistry, vol. 3 (eds. H. D. Holland and K. K. Turekian). Elsevier-Pergamon.

Sack R. O., Azeredo W. J. and Lipschutz M. E. (1991) Olivine diogenites - the mantle of the eucrite parent body. Geochim. Cosmochim. Acta 55, 1111-1120.

Saunier G., Poitrasson F., Moine B., Gregoire M. and Seddiki A. (2010) Effect of hot desert weathering on the bulk-rock iron isotope composition of L6 and H5 ordinary chondrites. Meteorit. Planet. Sci. 45, 195-209.

Schauble E. A., Rossman G. R. and Taylor H. P. (2001) Theoretical estimates of equilibrium Fe-isotope fractionations from vibrational spectroscopy. Geochim. Cosmochim. Acta 65, 2487-2497.

Schoenberg R. and von Blanckenburg F. (2006) Modes of planetary-scale $\mathrm{Fe}$ isotope fractionation. Earth Planet. Sci. Lett. 252, 342-359.

Schuessler J. A., Schoenberg R., Behrens H. and von Blanckenburg F. (2007) The experimental calibration of the iron isotope fractionation factor between pyrrhotite and peralkaline rhyolitic melt. Geochim. Cosmochim. Acta 71, 417-433.

Schuessler J. A., Schoenberg R. and Sigmarsson O. (2009) Iron and lithium isotope systematics of the Hekla volcano, Iceland evidence for $\mathrm{Fe}$ isotope fractionation during magma differentiation. Chem. Geol. 258, 78-91.

Schwartz J. M. and McCallum I. S. (2005) Comparative study of equilibrated and unequilibrated eucrites: subsolidus thermal histories of Haraiya and Pasamonte. Am. Mineral. 90, 18711886.

Scott E. R. D. and Bottke W. F. (2011) Impact histories of angrites, eucrites, and their parent bodies. Meteorit. Planet. Sci. 46, 1878-1887.

Scott E. R. D., Greenwood R. C., Franchi I. A. and Sanders I. S. (2009) Oxygen isotopic constraints on the origin and parent bodies of eucrites, diogenites, and howardites. Geochim. Cosmochim. Acta 73, 5835-5853.

Shahar A., Young E. D. and Manning C. E. (2008) Equilibrium high-temperature $\mathrm{Fe}$ isotope fractionation between fayalite and magnetite: an experimental calibration. Earth Planet. Sci. Lett. 268, 330-338.

Stolper E. (1977) Experimental petrology of eucritic meteorites. Geochim. Cosmochim. Acta 41, 587-611.

Strelow F. W. E. (1980) Improved separation of iron from copper and other elements by anion-exchange chromatography on a 4percent cross-linked resin with high-concentrations of hydrochloric-acid. Talanta 27, 727-732.

Takeda H., Ishii T., Miyamoto M. and Duke M. B. (1978) Crystallization of pyroxenes in lunar KREEP basalt 15386 and meteoritic basalts. Lunar Planet. Sci. Conf. 9, \#1157 (abstr.).

Teng F. Z., Dauphas N. and Helz R. T. (2008) Iron isotope fractionation during magmatic differentiation in Kilauea Iki Lava Lake. Science 320, 1620-1622.

Teng F.-Z., Dauphas N., Helz R. T., Gao S. and Huang S. (2011) Diffusion-driven magnesium and iron isotope fractionation in Hawaiian olivine. Earth Planet. Sci. Lett. 308, 317-324.

Tissot F. and Dauphas N. (2011) Development of high precision $238 \mathrm{U} / 235 \mathrm{U}$ ratio measurements for cosmochemical applications. Lunar Planet. Sci. Conf. 42, \#1082 (abstr.).

Treiman A. H., Lanzirotti A. and Xirouchakis D. (2004) Ancient water on asteroid 4 Vesta: evidence from a quartz veinlet in the Serra de Mage eucrite meteorite. Earth Planet. Sci. Lett. 219, 189-199.
Trilling D. E., Rivkin A. S., Stansberry J. A., Spahr T. B., Crudo R. A. and Davies J. K. (2007) Albedos and diameters of three Mars Trojan asteroids. Icarus 192, 442-447.

Wadhwa M. (2008) Redox conditions on small bodies, the Moon and Mars. In Oxygen in the Solar System (eds. G. J. MacPherson, D. W. Mittlefehldt, J. H. Jones and S. B. Simon), Reviews in Mineralogy \& Geochemistry 68, pp. 493-510.

Warren P. H., Jerde E. A. and Kallemeyn G. W. (1989) Lunar meteorites: siderophile element contents, and implications for the composition and origin of the Moon. Earth Planet. Sci. Lett. 91, 245-260.

Warren P. H., Jerde E. A., Migdisova L. F. and Yaroshevsky A. A. (1990) Pomozdino - an anomalous, high-MgO/FeO, yet REErich eucrite. Proc. Lunar Planet. Sci. Conf. 20, 281-297.

Warren P. H., Greenwood J. P., Richardson J. W., Rubin A. E. and Verish R. S. (2000) Geochemistry of Los Angeles, a Ferroan, La- and Th-rich Basalt from Mars. Lunar Planet. Sci. Conf. 31, \#2001 (abstr.).

Warren P. H., Kallemeyn G. W., Huber H., Ulff-Moller F. and Choe W. (2009) Siderophile and other geochemical constraints on mixing relationships among HED-meteoritic breccias. Geochim. Cosmochim. Acta 73, 5918-5943.

Weisberg M. K., McCoy T. J. and Krot A. N. (2006) Systematics and evaluation of meteorite classification. In Meteorites and the Early Solar System II (eds. D. S. Lauretta and H. Y. J. McSween). University of Arizona Press.

Weyer S. and Ionov D. A. (2007) Partial melting and melt percolation in the mantle: the message from $\mathrm{Fe}$ isotopes. Earth Planet. Sci. Lett. 259, 119-133.

Weyer S. and Schwieters J. B. (2003) High precision Fe isotope measurements with high mass resolution MC-ICPMS. Int. J. Mass Spectrom. 226, 355-368.

Weyer S. and Seitz H. M. (2012) Coupled lithium- and iron isotope fractionation during magmatic differentiation. Chem. Geol. 294-295, 42-50.

Weyer S., Anbar A. D., Brey G. P., Munker C., Mezger K. and Woodland A. B. (2005) Iron isotope fractionation during planetary differentiation. Earth Planet. Sci. Lett. 240, 251-264.

Weyer S., Anbar A. D., Brey G. P., Munker C., Mezger K. and Woodland A. B. (2007) Fe-isotope fractionation during partial melting on Earth and the current view on the Fe-isotope budgets of the planets - (reply to the comment of F. Poitrasson and to the comment of B.L. Beard and C.M. Johnson on "Iron isotope fractionation during planetary differentiation" by S. Weyer, A.D. Anbar, G.P. Brey, C. Munker, K. Mezger and A.B. Woodland). Earth Planet. Sci. Lett. 256, 638-646.

Williams H. M., McCammon C. A., Peslier A. H., Halliday A. N., Teutsch N., Levasseur S. and Burg J. P. (2004) Iron isotope fractionation and the oxygen fugacity of the mantle. Science 304, 1656-1659.

Williams H. M., Markowski A., Quitte G., Halliday A. N., Teutsch N. and Levasseur S. (2006) Fe isotope fractionation in iron meteorites: new insights into metal-sulphide segregation and planetary accretion. Earth Planet. Sci. Lett. 250, 486-500.

Yamaguchi A., Barrat J. A., Greenwood R. C., Shirai N., Okamoto C., Setoyanagi T., Ebihara M., Franchi I. A. and Bohn M. (2009) Crustal partial melting on Vesta: evidence from highly metamorphosed eucrites. Geochim. Cosmochim. Acta 73, $7162-7182$.

Zhu X. K., Guo Y., O’Nions R. K., Young E. D. and Ash R. D. (2001) Isotopic homogeneity of iron in the early solar nebula. Nature 412, 311-313. 